\title{
Spacecraft Rendezvous by Differential Drag under
}

\section{Uncertainties}

\author{
Leonel Mazal ${ }^{1}$, David Pérez ${ }^{2}$, and Riccardo Bevilacqua ${ }^{3}$ \\ University of Florida, Gainesville, Florida, 32611 \\ Fabio Curti ${ }^{4}$ \\ University of Rome "Sapienza", Rome, Italy.
}

At Low Earth Orbits differentials in the drag force between spacecraft can be used for controlling their relative motion in the orbital plane. Current methods for determining the drag force may result in errors due to inaccuracies in the density models and drag coefficients. In this work, a methodology for relative maneuvering of spacecraft based on differential drag, accounting for uncertainties in the drag model is proposed. A dynamical model composed of the mean semi-major axis and argument of latitude is utilized for describing long range maneuvers. For this model, a Linear Quadratic Regulator is implemented, accounting for the uncertainties in the drag force. The actuation is the pitch angle of the satellites, considering saturation. The control scheme guarantees asymptotic stability of the system up to a certain magnitude of the state vector, which is determined by the uncertainties. Numerical simulations show that the method exhibits consistent robustness to accomplish the maneuvers, even in presence of realistic modeling of density fields, drag coefficients, the co-rotation of the atmosphere and zonal harmonics up to $J_{8}$.

\footnotetext{
${ }^{1}$ Postdoctoral Associate, Mechanical and Aerospace Engineering, University of Florida, MAE-A Building, Room 211, 32611, AIAA Professional Member

2 Postdoctoral Associate, Mechanical and Aerospace Engineering, University of Florida, MAE-A Building, Room 211, 32611, AIAA Professional Member.

3 Associate Prof., Mechanical and Aerospace Engineering, University of Florida, MAE-A Building, Room 308, 32611, AIAA Senior Member.

${ }^{4}$ Associate Prof., School of Aerospace Engineering, University of Rome "Sapienza", AIAA Senior Member.
} 


\section{Nomenclature}

$\bar{a} \quad=$ mean semi-major axis $[\mathrm{km}]$

$\mathbf{a}_{\mathrm{D}}=$ drag force per unit mass $\left[\mathrm{km} / \mathrm{s}^{2}\right]$

$A_{i}=$ Cross-sectional area of the surface $i$ of the spacecraft $\left[\mathrm{m}^{2}\right]$

$C_{B}=$ Spacecraft's ballistic coefficient $\left[\mathrm{m}^{2} / \mathrm{kg}\right]$

$C_{D_{i}}=$ Drag coefficient of the surface $i$ of the spacecraft

$\bar{i} \quad=$ mean inclination $[\mathrm{rad}]$

$m \quad=$ Spacecraft's mass $[\mathrm{kg}]$

$\mathbf{P}=$ Positive definite matrix, solution of the algebraic Riccati Equation

$\mathbf{r}=$ Spacecraft's inertial position $[\mathrm{km}]$

$S_{i}=$ Surface of the face $i$ of the spacecraft $\left[\mathrm{m}^{2}\right]$

$\mathbf{v}=$ Spacecraft's inertial velocity $[\mathrm{km} / \mathrm{s}]$

$\mathbf{v}_{\text {atm }}=$ Earth Atmosphere's inertial velocity $[\mathrm{km} / \mathrm{s}]$

$\mathbf{v}_{\text {rel }}=$ Velocity vector of the spacecraft relative to the atmosphere $[\mathrm{km} / \mathrm{s}]$

$\beta=$ attitude angle [deg]

$\eta \quad=$ uncertainties (modeling errors) in the input $[1 / \mathrm{km}]$

$\mu \quad=$ Earth's gravitational parameter $\left[\mathrm{km}^{3} / \mathrm{s}^{2}\right]$

$\nu \quad=$ Control input to the system $[1 / \mathrm{km}]$

$\bar{\theta} \quad=$ argument of latitude $[\mathrm{rad}]$

$\rho \quad=$ Earth's atmospheric density $\left[\mathrm{kg} / \mathrm{m}^{3}\right]$

\section{Introduction}

In low Earth's orbits (LEO), the drag force constitutes one of the main perturbations affecting satellite dynamics. The specific force (force per unit mass) $\mathbf{a}_{\mathrm{D}}$ generated by the drag force is usually modeled as [1, Page 549]

$$
\mathbf{a}_{\mathrm{D}}=-\rho \mathbf{v}_{\text {rel }}\left\|\mathbf{v}_{\text {rel }}\right\| C_{B}
$$


where $\mathbf{v}_{\text {rel }}$ denotes the velocity vector of the satellite relative to the atmosphere, $\rho$ represents the atmospheric density, and $C_{B}$ is the ballistic coefficient. The ballistic coefficient is defined as

$$
C_{B} \triangleq \frac{1}{2 m} \sum_{i}^{N} C_{D_{i}} A_{i}
$$

where $A_{i}$ stands for the cross-sectional area of the $i^{\text {th }}$ surface impinged by the particles, $C_{D_{i}}$ is the corresponding drag coefficient, and $m$ denotes the mass of the spacecraft. Equation (1) shows that $\mathbf{a}_{\mathrm{D}}$ acts always in the direction opposed to the vector $\mathbf{v}_{\text {rel }}$, which represents the inertial velocity of the satellite relative to the atmosphere. Since the atmosphere inertial velocity is usually a small component compared to the inertial velocity of a LEO satellite, it is frequently neglected for theoretical developments, leading to use the inertial velocity of the satellite $\mathbf{v}$ instead of $\mathbf{v}_{\text {rel }}$ in Eq. (1). Under this assumption, drag forces cannot have components perpendicular to the instantaneous plane of motion. This is a significant limitation for the use of drag to maneuver. Yet, within the plane of motion, certain relative maneuvers can be achieved by use of drag only, reducing the propellant needs in certain missions $[2,3]$.

The main effect of the drag force is reducing the semi-major axis and eccentricity of the orbit. If the variables of Eq. (1) are judiciously exploited, relative accelerations between two or more satellite can be generated, such that they are steered towards relative states desirable for specific multiple-satellite applications. This idea is usually termed differential-drag (DD) maneuvering. In recent years, the use of $\mathrm{DD}$ for satellite relative maneuvers has been actively investigated due to its potential for reducing the propellant needs in formation flying and cluster flight missions. Yet, one should keep in mind that DD maneuvers might increase the orbital decay of the satellites, if the implemented controllers require high values of $\mathbf{a}_{\mathrm{D}}$. Other factors limiting the use of differential drag are the vanishing effects of the drag force at altitudes above $600 \mathrm{~km}$, and the time required to conduct a maneuver, which could be weeks or months.

Leonard et. al. $[4,5]$ derived a control scheme that utilizes drag plates acting at either maximum or minimum drag. To that end, the in-plane dynamics was modeled with the Clohessy-Wiltshire equations [6], and the density was assumed constant. Carter and Humi [7] derived linearized equa- 
tions of relative motion that include effects caused by drag, assuming a drag force model proportional to the square of the velocity. Kumar and Ng [3] extended the work by Leonard et. al. to consider other acting perturbations, erroneous measurements and inter-satellite distances slightly larger than those considered by Leonard et. al., but still in the order of magnitude of few tens of kilometers.

Bevilacqua et. al. [8, 9] utilized the linear formulation obtained by Schweighart and Sedwick [10] to derive a DD-based control algorithm for steering the in-plane relative coordinates to zero. They assumed a constant density, and actuation provided by drag plates with two possible configurations: parallel or perpendicular to the the velocity vector, thus providing minimum or maximum drag force respectively. Based on Schweighart-Sedwick equations [10] and the same aforementioned drag-plates actuation, Pérez and Bevilacqua [11] removed the assumption on constant atmospheric density and proposed an adaptive Lyapunov based controller to perform rendezvous between two spacecraft. Moreover, an analytical expression for a critical value of the relative acceleration due to drag was proposed. The relative acceleration due to drag must be above this value to ensure convergence of the maneuver. Ben-Yaacov and Gurfil used DD to perform relative maneuvers for cluster-keeping purposes [12] and developed a controller based on the nonlinear dynamics of the Relative Orbital Elements (ROE) [13]. Linearized equations of motion of ROE accounting for secular $J_{2}$ effects and DD were derived by Schaub in [14]. Harris and Açıkmeşe [15] applied Optimal control for DD based maneuvers. Dell'Elce and Kerschen [16] utilize pseudospectral methods and model predictive control for planning and effectuating rendezvous maneuvers. The control input is a torque exerted onto a reaction wheel that modifies the attitude of the satellite, thereby modifying the acceleration due to drag exerted onto the satellites. Moreover, Dell'Elce et. al. $[17,18]$ proposed a robust optimal control approach for DD based rendezvous maneuvers.

So far, the research in this area has been mainly oriented to close-proximity maneuvers, whereas the potential of differential-drag based maneuvers can go beyond close-proximity operations. Indeed, the ORBCOMM constellation [19] utilizes differential drag, in an open-loop manner, to control the relative phase angles of the satellites. Moreover, Finley et al. [2] proposed to use DD to separate in phase a number of satellites which are initially in close proximity. One could also envision applications that require to guide satellites, which are initially separated by large distances (order 
of magnitude of $\sim 1000 \mathrm{~km}$ ) in the same orbital plane, along trajectories that drive them into close-proximity configurations. For this purpose, DD can be also utilized, enabling reductions in the propellant requirements.

One of the main difficulties of designing differential-drag maneuvers is the inherent uncertainties existing in some of the quantities in Eq. (1). The models of the Earth's atmospheric density field, as well as the drag coefficient values associated to various satellite geometries can be inaccurate [20], leading to uncertainties in the effects of differential-drag based maneuvers. Hence, when designing differential-drag based maneuvers these uncertainties should be accounted for.

One of the goals of this work is to design differential-drag cooperative maneuvers explicitly considering uncertainties in the drag models, which at the best knowledge of the authors has not received much attention. These maneuvers are aimed at steering the satellites from given initial conditions, to close-proximity configurations oriented to a rendezvous. Inspired by the flourishing Cube-sat format, this work assumes that the satellite geometries are rectangular parallelepipeds that can change their pitch angles in a continuous manner, hence varying the cross-sectional area and the resulting differential-drag accelerations.

The proposed approach aims at dealing with the uncertainties in the atmospheric density models and in the ballistic coefficients. To derive the control laws of the maneuvers, the problem is formulated using a linearized relative motion representation, based on orbital elements. The linearization is based on the main assumption that the difference in mean semi-major axes between the two satellites is small compared to the mean semi-major axes values. Unlike other linearized formulations $[6,10]$, this model allows large distances between the satellites, as long as both mean semi-major axes are kept close one to each other. In this manner, one can consider initially large phase-separations between the satellites, and drive them into close-proximity configurations. In this context, a Linear Quadratic Regulator (LQR) is proposed. Moreover, an analysis of the convergence of the system driven by the proposed LQR controller in the presence of bounded uncertainties is presented. This leads to determine gains ensuring that the system still converges, under these uncertainties, up to a certain norm of the state vector. Since the cross-sectional area of the satellites is limited, an assessment of the system under saturation is provided, showing that convergence is 
still achieved.

The approach presented is tested using maneuver simulations that include: space and time varying density using NRLMSISE-00 including the influence of the solar and geomagnetic activity, the effects of the rotation of atmosphere on the drag force, a realistic model for the drag coefficient which takes into account the composition of the surfaces, their orientation, and the thermal difference between the surfaces and the atmosphere, and a gravitational geopotential due to zonal harmonics up to $J_{8}$. The effects of these factors on the resulting maneuvers are studied by running different scenarios with different combinations of these factors.

The advancements on the state of the art in this work are:

- Development of an LQR approach for DD based maneuvering which guarantees convergence up to a bound around the desired final state under uncertainties in the density and the drag coefficient.

- For the developed dynamical model, the control law is augmented with a saturation function, still providing convergence.

- Validation of the LQR approach using numerical simulations for a long range re-phasing maneuver, including realistic density, variable drag coefficient, co-rotating atmosphere, and zonal harmonics up to $J_{8}$.

The remainder of the paper is organized as follows: Section II introduces the geometrical setup assumed to tackle the problem; Section III addresses the problem with a formulation based on orbital elements, deriving an LQR controller; Section IV shows numerical simulations supporting the theoretical statements. Finally, concluding remarks are provided in Section V.

\section{Description of the Problem}

Let two satellites, Chaser and Target, be in coplanar circular orbits. The main goal of this work is to derive DD based, closed-loop controllers that steer the satellites to an encounter. These maneuvers will be performed by varying the drag force generated on either satellite, with no thrust usage. 

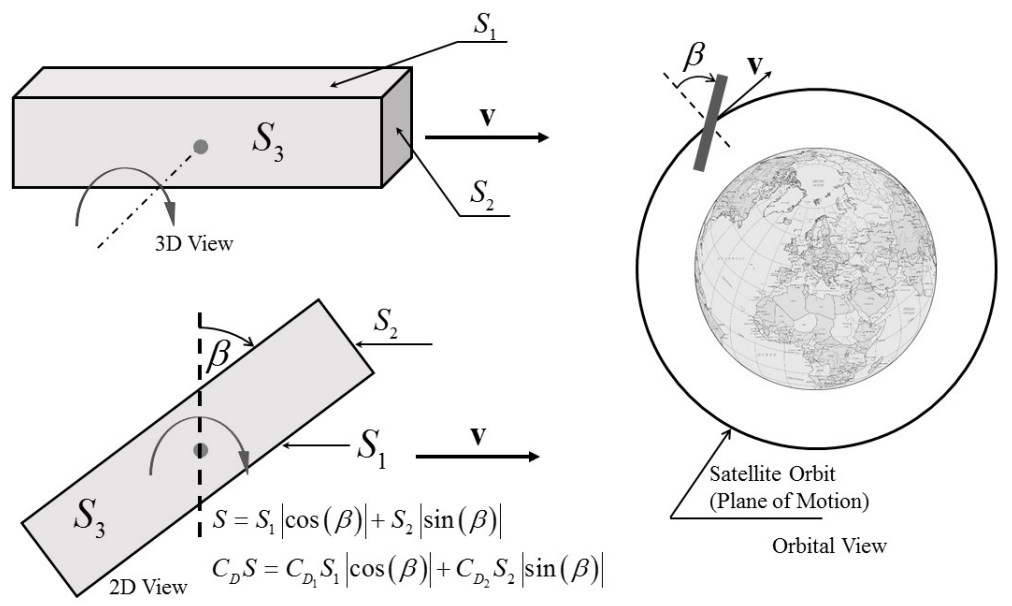

Fig. 1 Assumed geometry of the satellite.

Motivated by the rapid increase in the number of missions composed of Cube-sats, this work assumes that the satellite geometries are rectangular parallelepipeds, as the one illustrated in the 3D View of Figure 1. In this paper, these bodies are endowed with one rotational degree of freedom, being the axis of rotation always perpendicular to the plane of motion and depicted as a "dash-dot" line in the 3D View of Fig. 1. The input considered for the control laws is the attitude of the satellite parameterized by the angle $\beta$, according to Fig. 1 . To measure $\beta$, define a line lying on the orbital plane, perpendicular to the inertial velocity vector of the satellite $\mathbf{v}$, as the dashed line illustrated in the 2D View and Orbital View of Fig. 1. $\beta$ is measured from the aforementioned line towards the satellite velocity vector. In Fig. $1, S_{1}$ and $S_{2}$ denote the surfaces perpendicular to the plane of motion, whereas the face $S_{3}$ remains parallel to the plane of motion. For the forthcoming analytical developments, the velocity of the atmosphere is neglected, but it will then be incorporated in numerical simulations to assess the effects on the developed controller. Hence, the total product of the drag coefficient by cross-sectional area is given by

$$
\sum_{i=1}^{2} S_{i} C_{D_{i}}=C_{D_{1}} S_{1}|\cos \beta|+C_{D_{2}} S_{2}|\sin \beta|
$$

Changing $\beta$ modifies the cross-sectional areas and consequently the magnitude of the exerted acceleration $\mathbf{a}_{\mathrm{D}}$. Due to the periodicity of the cross-sectional areas with $\beta$, for the purposes of this work 
$\beta$ can be restricted to the range $\beta \in\left[0^{\circ}, 90^{\circ}\right]$, which allows to remove the absolute value operator from Eq. (3).

Because of the general lack of knowledge on the drag coefficient behaviours, for the purpose of the controller development the drag coefficient will be assumed to be a constant value, and equal for both satellites since the geometries are alike. However, since a constant value might be an erroneous modeling, the effects on the dynamics due to uncertain variations of the real values with respect to the assumed constant values will be theoretically assessed. Hence, assuming $C_{D_{i}}=C_{D}$ and constant, Equation (3) can be reformulated as

$$
\sum_{i=1}^{2} C_{D_{i}} S_{i}=C_{D} S=C_{D} S_{0} \cos (\beta-\psi)
$$

where $S_{0}=\sqrt{S_{1}^{2}+S_{2}^{2}}$ and $\psi=\arctan \left(\frac{S_{2}}{S_{1}}\right)$. It can be seen that $\sum_{i=1}^{2} C_{D_{i}} S_{i}$ has a maximum at $\beta=\psi>0$, and a minimum at $\beta=90^{\circ}$. Hence, for the purposes of this work, the range of $\beta$ can be restricted even more: $\beta \in\left[\psi, 90^{\circ}\right]$.

\section{Re-phasing Maneuvers under Uncertainties}

This section presents an approach to drive two satellites that are initially in circular orbits, in the same orbital plane but separated in phase, i.e. with different arguments of latitude, towards a close-proximity configuration. This configuration is attained by matching the mean semi-major axes $\bar{a}$ of the satellites and the mean argument of latitude $\bar{\theta}$. Notice that for initially circular orbits (very low eccentricities), neither the Earth's oblateness nor drag effects increase the mean eccentricities, i.e. the orbits will remain circular (in fact, drag reduces the eccentricity of orbits [1, Page 671]). Hence, matching $\bar{a}$ and $\bar{\theta}$ brings the two satellites into a close-proximity configuration.

\section{A. Dynamic Model}

Let $\bar{a}, \bar{e}, \bar{i}, \bar{\omega}$, and $\bar{M}$ respectively denote the mean semi-major axis, eccentricity, inclination, argument of perigee, and mean anomaly. Under the influence of drag and the first term of the gravitational geopotential due to zonal harmonics $\left(J_{2}\right)$, the time-variation of the mean argument of 
perigee $\bar{\omega}$ and the mean mean anomaly $\bar{M}$ are respectively given by [21] :

$$
\begin{gathered}
\dot{\bar{\omega}}=\frac{3}{4} J_{2} \bar{n}\left(\frac{R_{e q}}{\bar{p}}\right)^{2}\left(5 \cos ^{2} \bar{i}-1\right) \\
\dot{\bar{M}}=\bar{n}+\frac{3}{4} J_{2} \bar{n}\left(\frac{R_{e q}}{\bar{p}}\right)^{2} \sqrt{1-\bar{e}^{2}}\left(3 \cos ^{2} \bar{i}-1\right)
\end{gathered}
$$

where $\bar{p}$ and $\bar{n}$ denote the parameter (semilatus rectum) of the orbits and the mean motion, respectively, and $R_{e q}$ represents the mean equatorial radius of the Earth.

The argument of latitude $\theta$ is defined as $\theta \triangleq \omega+f$, where $f$ denotes the true anomaly. Assuming circular orbits, the variation of the mean argument of latitude can be modeled as

$$
\begin{aligned}
\dot{\bar{\theta}} & =\dot{\bar{M}}+\dot{\bar{\omega}} \\
& =\sqrt{\frac{\mu}{\bar{a}^{3}}}+\frac{3}{4} J_{2} \sqrt{\frac{\mu}{\bar{a}^{3}}}\left(\frac{R_{e q}}{\bar{a}}\right)^{2}\left(8 \cos ^{2} \bar{i}-2\right)
\end{aligned}
$$

To model the rate of change of the mean semi-major axis, we use the Gauss variational equations (GVE) and the premise that "the effects of the control vector $\mathbf{u}$ is assumed to have the same effect on the mean orbit elements as it has on the osculating orbit elements" as mentioned by Schaub and Alfriend [22]. In this work, the vector $\mathbf{u}$ will be generated using drag forces. Hence, the effects of the drag on the mean semi-major axis will be approximated by the effect that the same drag would generate on the corresponding osculating semi-major axis. This approximation has been proposed and assessed in a few papers $[21,23,24]$ showing its validity.

Using the GVE resolved in tangential and normal axes [25, Page 489], the time-variation of the semi-major axis $a$ is formulated as

$$
\dot{a}=\frac{2 a^{2} v}{\mu} \Gamma_{t}
$$

where $v \triangleq\|\mathbf{v}\|$, and $\Gamma_{t}$ represents the disturbance acceleration component along the inertial velocity vector. The perturbation due to $J_{2}$ has no effect on $\bar{a}$. Neglecting any motion of the atmosphere 
and considering Eq. (4), the input $\Gamma_{t}$ due to drag is given by

$$
\Gamma_{t}=-\frac{1}{2} \rho v^{2} \frac{C_{D}}{m} S_{0} \cos (\beta-\psi)
$$

Since for circular orbits $v=\sqrt{\mu / a}$, introducing Eq. (9) into Eq. (8), and recalling that the rate of change of the mean semi-major axis due to drag will be approximated by the rate of change that the drag would induce onto the osculating semi-major axis [22], $\dot{\bar{a}}$ is approximated by

$$
\dot{\bar{a}}=-2 \sqrt{\mu \bar{a}} \rho \frac{C_{D} S_{0}}{2 m} \cos (\beta-\psi)
$$

The quantity $C_{D} S_{0} /(2 \mathrm{~m})$ will be denoted by $C_{B 0}$ and will be considered constant for the development of the control law. However, in reality, the real drag coefficient is affected by variations of the attitude, temperature and chemical composition of the surface of the spacecraft and the environment [26-28]. The effects of these uncertain variations in the performance of the control law will be addressed in Section III C.

Recall that under $J_{2}$ and drag influence, the mean inclination $\bar{i}$ remains constant, and thus it actually represents a parameter. It is important to mention that the reduced state $[\bar{\theta}, \bar{a}]^{\top}$ represents only the in-plane motion.

The relative orbital elements are defined as:

$$
\Delta \bar{\theta} \triangleq \bar{\theta}_{C}-\bar{\theta}_{T}
$$

$$
\Delta \bar{a} \triangleq \bar{a}_{C}-\bar{a}_{T}
$$

In Eqs. (11) and (12), as well as for the remainder of the paper, the sub-indices $(\cdot)_{C}$ or $(\cdot)_{T}$ refer to the parameter or variable $(\cdot)$ associated to the Chaser or Target respectively. The lack of sub-index in certain expressions indicates that the expression is valid for either spacecraft indistinctly.

Generally speaking, in multiple-satellite missions that involve coordinated relative motion, the 
mean semi-major axes should be close on to each other, i.e. $\frac{\left|\bar{a}_{C}-\bar{a}_{T}\right|}{\bar{a}_{T}} \ll 1$; otherwise there would be high natural drift rates that could rapidly dismantle any desired configuration.

Consider a virtual satellite 0 , in a circular orbit, with constant mean semi-major axis $\bar{a}_{0}$ (i.e. unaffected by drag). Assume $\bar{a}_{0}$ is sufficiently close to $\bar{a}_{C}$ and $\bar{a}_{T}$, and the inclination $\bar{i}_{0}=\bar{i}_{C}=\bar{i}_{T}=$ $\bar{i}$. Considering Eq. (7), the difference in the rate of change of the arguments of latitude between the satellites $C$ and 0 can be linearized as

$$
\dot{\bar{\theta}}_{C}-\left.\dot{\overline{\theta_{0}}} \simeq \frac{\partial \dot{\bar{\theta}}}{\partial \bar{a}}\right|_{\bar{a}=\bar{a}_{0}}\left(\bar{a}_{C}-\bar{a}_{0}\right)
$$

The difference in the rate of change of the arguments of latitude between the satellites $T$ and 0 can be obtained in a similar manner. Finally,

$$
\Delta \dot{\bar{\theta}}=\dot{\bar{\theta}}_{C}-\dot{\bar{\theta}}_{0}-\left(\dot{\bar{\theta}}_{T}-\dot{\bar{\theta}}_{0}\right)=-P_{0} \Delta \bar{a}
$$

where

$$
P_{0} \triangleq \sqrt{\mu}\left[\frac{3}{2} \frac{1}{\bar{a}_{0}^{5 / 2}}+\frac{21}{8} \frac{J_{2} R_{e q}^{2}\left(8 \cos ^{2} \bar{i}-2\right)}{\bar{a}_{0}^{9 / 2}}\right]
$$

Eq. (14) represents the evolution of the relative mean argument of latitude, as a function of the mean relative semi-major axis. Due to the linearizations, this equation remains valid as long as the mean semi-major axes of Chaser and Target remain sufficiently close to $\bar{a}_{0}$. As previously stated, because of the coordinated motion, $\bar{a}_{C}$ and $\bar{a}_{T}$ are expected to remain relatively close one to each other; otherwise, there would be a high drift hindering any possible coordinated maneuver. Moreover, during the maneuvers, the variations of the mean semi-major axes due to drag are expected to be sufficiently small holding the linearizations valid, i.e. $\frac{\left|\bar{a}(t)-\bar{a}_{0}\right|}{\bar{a}_{0}} \ll 1$. These assumptions will be validated during the extensive numerical simulations, presented in Section IV.

To obtain $\Delta \dot{\bar{a}}$, considering Eq. (10), and taking the $0^{\text {th }}$ order Taylor expansion about $\bar{a}=\bar{a}_{0}$, 
yields

$$
\Delta \dot{\bar{a}}=-2 \sqrt{\mu \bar{a}_{0}}\left(\rho_{C} u_{C}-\rho_{T} u_{T}\right)
$$

where

$$
u_{C} \triangleq C_{B 0} \cos \left(\beta_{C}-\psi\right) \quad u_{T} \triangleq C_{B 0} \cos \left(\beta_{T}-\psi\right)
$$

Defining $\mathbf{w} \triangleq[\Delta \bar{\theta}, \Delta \bar{a}]^{\top}$, the obtained linear system can be written as follows

$$
\dot{\mathbf{w}}=\left[\begin{array}{cc}
0 & -P_{0} \\
0 & 0
\end{array}\right] \mathbf{w}+\left[\begin{array}{l}
0 \\
b
\end{array}\right]\left(-\rho_{C} u_{C}+\rho_{T} u_{T}\right)
$$

where $b \triangleq 2 \sqrt{\mu \bar{a}_{0}}$. The dynamical system (18) may be considered linear time-invariant (LTI). The input of this system is given by the term $-\rho_{C} u_{C}+\rho_{T} u_{T}$. Notice that the uncertainties in the densities $\rho_{C}$ and $\rho_{T}$, and in the drag coefficient $C_{D}$, constitute uncertainties in the input.

\section{B. Controller Derivation}

Assuming no constraints on the input, an infinite-horizon LQR approach will be initially implemented. Since the range of $\nu \triangleq-\rho_{C} u_{C}+\rho_{T} u_{T}$ is limited, a saturation function will be proposed in the sequel, and it will be shown that convergence is still achieved. Recall that, in an infinite-horizon $\mathrm{LQR}$, if $\mathbf{w}$ denotes the state vector, the input is given by [29, Chapter 3.3]

$$
\boldsymbol{\nu}_{L Q R}=-R^{-1} \mathbf{B}^{\top} \mathbf{P} \mathbf{w}
$$

where $\mathbf{P}$ is the matrix that solves the algebraic Riccati Equation formulated as

$$
\mathbf{A}^{\top} \mathbf{P}+\mathbf{P} \mathbf{A}-\mathbf{P} \mathbf{B} R^{-1} \mathbf{B}^{\top} \mathbf{P}+\mathbf{Q}=\mathbf{O}
$$


For the problem in question, the matrices are given by

$$
\begin{aligned}
& \text { - } \mathbf{A}=\left[\begin{array}{ll}
0 & -P_{0} \\
0 & 0
\end{array}\right], \\
& \text { - } \mathbf{B}=\left[\begin{array}{l}
0 \\
b
\end{array}\right],
\end{aligned}
$$

- $R>0$,

- $\mathbf{Q}$ is a positive definite matrix defined as $\mathbf{Q} \triangleq\left[\begin{array}{ll}q_{1} & 0 \\ 0 & q_{2}\end{array}\right]$, i.e. $q_{1}>0$ and $q_{2}>0$,

- $\mathbf{P}$ is a positive definite matrix defined as $\mathbf{P} \triangleq\left[\begin{array}{ll}\Pi_{1} & \Pi_{2} \\ \Pi_{2} & \Pi_{3}\end{array}\right]$,

$$
\boldsymbol{-} \mathbf{O} \triangleq\left[\begin{array}{ll}
0 & 0 \\
0 & 0
\end{array}\right]
$$

For the discussed problem, $\mathbf{P}$ is sought such that the matrix

$$
\mathbf{A}^{*} \triangleq \mathbf{A}-\mathbf{B} R^{-1} \mathbf{B}^{\top} \mathbf{P}
$$

is Hurwitz, i.e. all the eigenvalues of $\mathbf{A}^{*}$ must have negative real part. Hence, the expressions for the entries of the matrix $\mathbf{P}$ are determined as

$$
\begin{aligned}
& \Pi_{1}=\frac{\sqrt{q_{1}} \sqrt{2 P_{0} \sqrt{q_{1} R+q_{2} b}}}{P_{0} \sqrt{b}} \\
& \Pi_{2}=-\frac{\sqrt{q_{1} R}}{b} \\
& \Pi_{3}=\frac{\sqrt{R} \sqrt{2 P_{0} \sqrt{q_{1} R}+q_{2} b}}{b^{3 / 2}}
\end{aligned}
$$

and the gain $\mathbf{K}$ is

$$
\mathbf{K}=R^{-1} \mathbf{B}^{\top} \mathbf{P}=\left[-\sqrt{\frac{q_{1}}{R}} \sqrt{2 \frac{P_{0}}{b} \sqrt{\frac{q_{1}}{R}}+\frac{q_{2}}{b}}\right]
$$


With $\mathbf{K}$ already computed, then the desired input to the system is

$$
\nu_{\mathrm{des}}=-\mathbf{K}[\Delta \bar{\theta}, \Delta \bar{a}]^{\top}
$$

Now, assuming that

$$
\left(-\rho_{C}+\rho_{T} \zeta\right) C_{B 0} \leq \nu_{\mathrm{des}} \leq\left(-\rho_{C} \zeta+\rho_{T}\right) C_{B 0}
$$

where $\zeta \triangleq \cos (\pi / 2-\psi)$, one should find $\beta_{C}$ and $\beta_{T}$ (the pitch angles of the Chaser and Target, respectively), such that

$$
-\rho_{C} u_{C}+\rho_{T} u_{T}=\nu_{\mathrm{des}}
$$

If the constraints (27) are not satisfied, there are no $\beta_{C} \in \mathbb{R}$ and $\beta_{T} \in \mathbb{R}$ that satisfy Eq. (28). This case will be addressed in the sequel.

\section{Effects of the Uncertainties in the Implementation}

In order to find $\beta_{C}$ and $\beta_{T}$ that solve Eq. (28), a model for the atmospheric density must be selected, from which the densities for the Chaser and Target are respectively assumed to be $\rho_{C}^{*}, \rho_{T}^{*}$. Similarly, one should also consider a nominal value for the drag coefficient $C_{D}^{*}$, which leads to a nominal value $C_{B 0}^{*}=S_{0} C_{D}^{*} /(2 \mathrm{~m})$, yielding assumed $u_{C}^{*}=C_{B 0}^{*} \cos \left(\beta_{C}-\psi\right)$ and $u_{T}^{*}=$ $C_{B 0}^{*} \cos \left(\beta_{T}-\psi\right)$. Hence, if

$$
\left(-\rho_{C}^{*}+\rho_{T}^{*} \zeta\right) C_{B 0}^{*} \leq \nu_{\mathrm{des}} \leq\left(-\rho_{C}^{*} \zeta+\rho_{T}^{*}\right) C_{B 0}^{*}
$$

$\beta_{C}$ and $\beta_{T}$ are actually found from the equation

$$
\left(-\rho_{C}^{*} u_{C}^{*}+\rho_{T}^{*} u_{T}^{*}\right)=\nu_{\mathrm{des}}
$$


However, considering that the models are uncertain, the real densities affecting the spacecraft are given by $\rho_{C}=\rho_{C}^{*}+\delta \rho_{C}$ and $\rho_{T}=\rho_{T}^{*}+\delta \rho_{T}$, where $\delta \rho_{C}$ and $\delta \rho_{T}$ denote the differences between the real and assumed density, for Chaser and Target respectively. Correspondingly, the real value of $u_{C}$ and $u_{T}$ are given by $u_{C}=u_{C}^{*}+\delta u_{C}$ and $u_{T}=u_{T}^{*}+\delta u_{T}$.

Hence, the true input of the system is given by

$$
\begin{aligned}
\nu_{\text {true }} & =-\left(\rho_{C}^{*}+\delta \rho_{C}\right)\left(u_{C}^{*}+\delta u_{C}\right)+\left(\rho_{T}^{*}+\delta \rho_{T}\right)\left(u_{T}^{*}+\delta u_{T}\right) \\
& =\nu_{\text {des }}+\eta
\end{aligned}
$$

where

$$
\eta \triangleq-\rho_{C}^{*} \delta u_{C}+\rho_{T}^{*} \delta u_{T}-\delta \rho_{C} u_{C}^{*}+\delta \rho_{T} u_{T}^{*}-\delta \rho_{C} \delta u_{C}+\delta \rho_{T} \delta u_{T}
$$

For the forthcoming analysis, the respective quantities are considered normalized by the corresponding units; i.e. distances by $1 \mathrm{~km}$, angles by $1 \mathrm{rad}$, mass by $1 \mathrm{~kg}$, and time by 1 second. To assess the effects on the maneuvers caused by the errors in the density models and drag coefficient, consider the positive definite function $V=\mathbf{w}^{\top} \mathbf{P} \mathbf{w}$. Considering Eq. (18), its time derivative is then given by

$$
\dot{V}=2 \mathbf{w}^{\top} \mathbf{P}\left[\mathbf{A} \mathbf{w}+\mathbf{B}\left(-\left(\rho_{C}^{*}+\delta \rho_{C}\right)\left(u_{C}^{*}+\delta u_{C}\right)+\left(\rho_{T}^{*}+\delta \rho_{T}\right)\left(u_{T}^{*}+\delta u_{T}\right)\right)\right]
$$

Introducing Eqs. (26), (30) and (32), yields

$$
\begin{aligned}
\dot{V} & =2 \mathbf{w}^{\top} \mathbf{P}\left[\mathbf{A} \mathbf{w}+\mathbf{B}\left(\nu_{\mathrm{des}}+\eta\right)\right] \\
& =2 \mathbf{w}^{\top} \mathbf{P}[(\mathbf{A}-\mathbf{B} \mathbf{K}) \mathbf{w}+\mathbf{B} \eta] \\
& =\mathbf{w}^{\top}\left[\mathbf{P}(\mathbf{A}-\mathbf{B} \mathbf{K})+(\mathbf{A}-\mathbf{B} \mathbf{K})^{\top} \mathbf{P}\right] \mathbf{w}+2 \mathbf{w}^{\top} \mathbf{P B} \eta \\
& =-\mathbf{w}^{\top}\left(\mathbf{Q}+\mathbf{P B} R^{-1} \mathbf{B}^{\top} \mathbf{P}\right) \mathbf{w}+2 \mathbf{w}^{\top} \mathbf{P B} \eta
\end{aligned}
$$


which can be upper bounded by

$$
\dot{V} \leq \Upsilon(\|\mathbf{w}\|) \triangleq-\|\mathbf{w}\|^{2} \lambda_{\min }+2\|\mathbf{w}\|\|\mathbf{P B}\| \bar{\eta}
$$

where $\bar{\eta} \geq|\eta|, \lambda_{\min }>0$ denotes the smallest eigenvalue of the symmetric matrix $\boldsymbol{\Xi} \triangleq \mathbf{Q}+$ $\mathbf{P B} R^{-1} \mathbf{B}^{\top} \mathbf{P}$. Based on Eq. (32), an upper bound for $|\eta|$ can be formulated as

$$
|\eta| \leq \bar{\eta} \triangleq 2\left(\rho^{* M} \delta u^{M}+u^{* M} \delta \rho^{M}+\delta \rho^{M} \delta u^{M}\right)
$$

where the supra-script $M$ indicates that the maximum possible value is taken for the corresponding parameter. Generally speaking, $\bar{\eta}$ could be computed based on the maximum expected errors for a given model of density and a given model of the ballistic coefficient. This will be discussed further in the sequel with specific models.

Considering the errors in the atmospheric density models and in the ballistic coefficients, $\dot{V}$ will be always bounded from above by $\Upsilon(\|\mathbf{w}\|)$, which is a parabola in $\|\mathbf{w}\|$. Its roots are located at $\|\mathbf{w}\|=0$ and $\|\mathbf{w}\|=2 \frac{\|\mathbf{P} \mathbf{B}\|}{\lambda_{\min }} \bar{\eta}>0$. Hence, as long as $\|\mathbf{w}\|>2 \frac{\|\mathbf{P} \mathbf{B}\|}{\lambda_{\min }} \bar{\eta}, \dot{V}$ will be negative, as required for convergence [30, Chapter 4]. Since $V$ is positive definite, due to continuity and as long as $\dot{V}<0$, eventually $\|\mathbf{w}\|$ becomes $2 \frac{\|\mathbf{P} \mathbf{B}\|}{\lambda_{\min }} \bar{\eta}$ and the decreasing rate of $V$ cannot be guaranteed. Hence, there is interest in reducing the ratio $\|\mathbf{P} \mathbf{B}\| / \lambda_{\min }$, which is a function of $\mathbf{Q}$ and $R$, to reduce the range in which $\dot{V}<0$ cannot be guaranteed.

The matrix $\boldsymbol{\Xi}=\mathbf{Q}+\mathbf{P B} R^{-1} \mathbf{B}^{\top} \mathbf{P}$ is given by

$$
\boldsymbol{\Xi}=\left[\begin{array}{ll}
\Xi_{1} & \Xi_{2} \\
\Xi_{2} & \Xi_{3}
\end{array}\right]
$$

where

$$
\text { - } \Xi_{1}=2 q_{1}
$$

- $\Xi_{2}=-\sqrt{\frac{q_{1}}{b}} \sqrt{2 P_{0} \sqrt{q_{1} R}+q_{2} b}$

- $\Xi_{3}=2 q 2+\frac{2 P_{0} \sqrt{q_{1} R}}{b}$ 
from which its eigenvalues are computed as:

$$
\lambda_{\text {max }, \text { min }}=\frac{q_{2} b+P_{0} \sqrt{q_{1} R}+q_{1} b \pm \sqrt{q_{2}^{2} b^{2}+2 q_{2} b P_{0} \sqrt{q_{1} R}-q_{1} q_{2} b^{2}+q_{1} R P_{0}^{2}+q_{1}^{2} b^{2}}}{b}
$$

Hence,

$$
\frac{\|\mathbf{P B}\|}{\lambda_{\min }}=\frac{\sqrt{\tilde{q}_{1}+\tilde{q}_{2}+2 \frac{P_{0}}{b} \sqrt{\tilde{q}_{1}}}}{\left(\tilde{q}_{1}+\tilde{q}_{2}\right)+\frac{P_{0}}{b} \sqrt{\tilde{q}_{1}}-\sqrt{\left(\tilde{q}_{1}^{2}+\tilde{q}_{2}^{2}\right)+\left(\frac{P_{0}}{b}\right)^{2} \tilde{q}_{1}+2 \frac{P_{0}}{b} \sqrt{\tilde{q}_{1}} \tilde{q}_{2}-\tilde{q}_{1} \tilde{q}_{2}}}
$$

where $\tilde{q}_{1} \triangleq q_{1} / R$ and $\tilde{q}_{2} \triangleq q_{2} / R$. Notice that the right-hand side of Eq. (39) does not depend on $q_{1}, q_{2}$ or $R$ explicitly, but on $\tilde{q}_{1}$ and $\tilde{q}_{2}$. Moreover, $\|\mathbf{P} \mathbf{B}\| / \lambda_{\min }$ is a positive function of $\tilde{q}_{1}$ and $\tilde{q}_{2}$, which can be selected to reduce $\|\mathbf{P} \mathbf{B}\| / \lambda_{\min }$ as much as desired, while keeping in mind that this affects the required control efforts and might generate saturation in the system. Yet, next section shows that if the system is saturated, it will eventually reach a non-saturated configuration where Eq. (29) is satisfied, and thus convergence up to $\|\mathbf{w}\|=2 \frac{\|\mathbf{P} \mathbf{B}\|}{\lambda_{\min }} \bar{\eta}>0$ can be achieved.

\section{Saturation}

According to the proposed controller, the desired input of the system is given by Eq. (26). On the other hand, the required attitude angles $\beta_{C}$ and $\beta_{T}$ are computed according to Eq. (30). As previously mentioned, in order to find $\beta_{C} \in \mathbb{R}$ and $\beta_{T} \in \mathbb{R}$, the inequality (29) must hold. However, for certain values of $\Delta \bar{\theta}$ and $\Delta \bar{a}$ the inequality (29) might be not satisfied, and thus there are no valid $\beta_{C}$ and $\beta_{T}$ satisfying Eqs. (26) and (30). This occurs because the ballistic coefficients of the satellites are limited, whereas the input of a linear controller of the form of Eq. (26) can, theoretically, attain any value.

To address this problem, this work proposes to implement a saturation function. Whenever the inequality (29) is not satisfied, the system implements attitudes $\beta_{C}$ and $\beta_{T}$ such that the maximum or minimum feasible differential drag acceleration is obtained. It yields, 


$$
\begin{array}{ll}
\beta_{C}=\frac{\pi}{2} \text { and } \beta_{T}=\psi, & \text { if }-\mathbf{K}[\Delta \bar{\theta}, \Delta \bar{a}]^{\top} \geq C_{B 0}\left(-\rho_{C}^{*} \zeta+\rho_{T}^{*}\right) \\
\beta_{C}=\psi \text { and } \beta_{T}=\frac{\pi}{2}, & \text { if }-\mathbf{K}[\Delta \bar{\theta}, \Delta \bar{a}]^{\top} \leq C_{B 0}\left(-\rho_{C}^{*}+\rho_{T}^{*} \zeta\right) \\
C_{B 0}^{*}\left(-\rho_{C}^{*} \cos \left(\beta_{C}-\psi\right)+\rho_{T}^{*} \cos \left(\beta_{T}-\psi\right)\right)=-\mathbf{K}[\Delta \bar{\theta}, \Delta \bar{a}]^{\top}, \text { otherwise }
\end{array}
$$

The goal of this section is showing that the dynamical system given by Eqs. (14) and (16) still converges to the origin, if it is driven by the control law stated by Eqs. (40-42). This will be done by depicting the phase portrait of the system and analysing the resulting trajectories.

Since the atmospheric density certainly depends on the altitude of the satellites, it is necessary to assume a density field model that captures the main density behavior due to changes in the semi-major axes of the satellites. Analytical expressions of the density as a function of the altitude show an exponential decay due to hydrostatic equilibrium. On the other hand, there exist variations of the density due to effects other than altitude, like solar activity, diurnal cycles, etc.. Yet, the simplest models tend to average out these variations, showing only variations due to altitude. The forthcoming analysis examines the behavior of the two satellites, under saturation, assuming that the main variations of the density are due to altitude, and any other effect is averaged out. Still, the effects due to the expected uncertainties on the dynamics will be addressed. Hence, for the forthcoming analysis, the exponential atmospheric model CIRA-72 published in [1, Page 564] will be used, as it captures the aforementioned behavior and allows to keep the math tractable. In this model, the atmospheric density is computed as

$$
\rho(h)=\rho_{H} e^{-\frac{h-h_{0}}{H}}
$$

where $\rho_{H}, h_{0}$, and $H$ denote model parameters that are tabulated in the aforementioned reference, for various intervals of altitude $h$.

Since the orbits are assumed circular (assuming spherical Earth with radius $R_{e q}$ ), Eq. (43) can 
be approximately reformulated as

$$
\rho(\bar{a})=\rho_{H} e^{-\frac{\bar{a}-\left(h_{0}+R_{e q}\right)}{H}}
$$

where the constant $h_{0}$ represents the lowest altitude of the interval of interest. Since the mean semi-major axes of the satellites are expected to be sufficiently close, from Eq. (44), $\rho_{C}^{*}$ can be modeled as

$$
\rho_{C}^{*} \simeq \rho_{T}^{*}+\left.\frac{\partial \rho}{\partial \bar{a}}\right|_{\bar{a}_{T}} \Delta \bar{a}=\rho_{T}^{*}\left(1-\frac{\Delta \bar{a}}{H}\right)
$$

where $H$ is selected for the proper range of altitudes. Any density variations due to solar activity would result in time-varying coefficients for the model (44). Yet, the same relation (45) would be obtained, provided that the semi-major axes of the satellites are sufficiently close. Moreover, notice that for a constant density model, Eq. (44) is still valid with $H$ that tends to infinity.

To proceed, the region in which Eq. (42) has solutions $\beta_{C} \in \mathbb{R}$ and $\beta_{T} \in \mathbb{R}$ is firstly determined. From the inequality (29) and introducing Eq. (45), this region is obtained as

$$
S_{U} \Delta \bar{\theta}+M_{U} \geq \Delta \bar{a} \geq M_{L}+S_{L} \Delta \bar{\theta}
$$

where

$$
\begin{gathered}
S_{U} \triangleq \frac{-k_{1}}{k_{2}+\frac{C_{B 0}^{*}}{H} \rho_{T}^{*}}>0 \\
S_{L} \triangleq \frac{-k_{1}}{k_{2}+\frac{C_{B 0}^{*}}{H} \zeta \rho_{T}^{*}}>0 \\
M_{U} \triangleq \frac{(1-\zeta) C_{B 0}}{\frac{k_{2}}{\rho_{T}^{*}}+\frac{C_{B 0}^{*}}{H}}>0
\end{gathered}
$$




$$
M_{L} \triangleq-\frac{C_{B 0}^{*}(1-\zeta)}{\frac{k_{2}}{\rho_{T}^{*}}+\frac{C_{B 0}}{H} \zeta}<0
$$

where $k_{1}$ and $k_{2}$ are the first and second components of the matrix $\mathbf{K}$, respectively. Notice that, for a given $\rho_{T}^{*}, \Delta \bar{a}$ is bounded by an upper line $\mathcal{L}_{U}$ and a lower line $\mathcal{L}_{L}$, of positive slopes. Figure 2 shows these lines (dash-dot) for arbitrary (but typical) values of the parameters of the inequality (46), namely: $C_{D}=2.2, C_{B 0}^{*}=0.0134 \mathrm{~m}^{2} / \mathrm{kg}, S_{1}=0.06 \mathrm{~m}^{2}$ and $S_{2}=0.01 \mathrm{~m}^{2}, m=5 \mathrm{~kg}$, $k_{1}=-1.830310^{-10} 1 / \mathrm{km}$ and $k_{2}=1.854210^{-10} 1 / \mathrm{km}^{2}, H=58.5150 \mathrm{~km} \mathrm{[1]}$, and $\rho_{T}^{*}=2.56310^{-12}$ $\mathrm{kg} / \mathrm{m}^{3}$, corresponding to an altitude of $421.87 \mathrm{~km}$ [1]. For the following explanation, the zone in between $\mathcal{L}_{U}$ and $\mathcal{L}_{L}$ will be referred to as non-saturated zone. Moreover, Fig. 2 also illustrates the solid line $\mathcal{O}$ that satisfies $\Delta \dot{\bar{a}}=0$. This line is obtained by solving $k_{1} \Delta \bar{\theta}+k_{2} \Delta \bar{a}=0$. The slope of this line is $-k_{1} / k_{2}>0$, and it passes through the origin. Above(below) $\mathcal{O}, \Delta \dot{\bar{a}}<(>) 0$. Furthermore, notice that above(below) the line $\Delta \bar{a}=0, \Delta \dot{\bar{\theta}}<(>) 0$.

According to Eq. (16), there are two necessary conditions that must hold in order to be able to generate differential drag for control purposes. These conditions are formulated as $-\rho_{C}^{*}+\rho_{T}^{*} \zeta<0$ and $-\rho_{C}^{*} \zeta+\rho_{T}^{*}>0$. If one of these conditions does not hold, $\Delta \dot{\bar{a}}$ could not be generated in both directions, positive and negative. Considering Eq. (45), these conditions entail the following requirements: $1>\zeta+\Delta \bar{a} / H$ and $1>\zeta(1-\Delta \bar{a} / H)$. As it was previously mentioned, the expected values of $\Delta \bar{a}$ are sufficiently small to avoid high natural drift rates, from which for the purposes of this work they can be assumed as $|\Delta \bar{a}| \leq 10 \mathrm{~km}$. Moreover, for 3U or longer Cube-sats, $\zeta=$ $\cos (\pi / 2-\psi) \leq 0.32$. Hence, $1>\zeta+\Delta \bar{a} / H$ and $1>\zeta(1-\Delta \bar{a} / H)$ do not appear difficult to satisfy. It means that from the viewpoint of the control law (assuming no uncertainties), the differential drag input, $\rho_{C}^{*} u_{C}^{*}+\rho_{T}^{*} u_{T}^{*}$, can be generated both positive and negative, as possibly required by the controller.

If these conditions were not satisfied, the non-saturated zone would not exist, nor the line $\mathcal{O}$ could be defined.

Figure 2 also depicts the phase portrait of the system (18) driven by (40-42). At each point $\mathbf{w}=[\Delta \bar{\theta}, \Delta \bar{a}]^{\top}$, the slope of the flow direction is computed as $\frac{\mathrm{d} \Delta \bar{a}}{\mathrm{~d} \Delta \bar{\theta}}=\Delta \dot{\bar{a}} / \Delta \dot{\bar{\theta}}$. In the non-saturated 
zone, the slope of the flow direction is given by

$$
\frac{\mathrm{d} \Delta \bar{a}}{\mathrm{~d} \Delta \bar{\theta}}=\frac{b}{P_{0}}\left(k_{1} \frac{\Delta \bar{\theta}}{\Delta \bar{a}}+k_{2}\right)
$$

whereas out of this region, the slopes are given by

$$
\begin{aligned}
\frac{\mathrm{d} \Delta \bar{a}}{\mathrm{~d} \Delta \bar{\theta}} & =-\frac{b}{P_{0} \Delta \bar{a}}\left(C_{B 0}^{*}\left(-\rho_{C}^{*}+\rho_{T}^{*} \zeta\right)\right) \\
& =-\frac{b C_{B 0}^{*} \rho_{T}^{*}\left(\zeta-\left(1-\frac{\Delta \bar{a}}{H}\right)\right)}{P_{0} \Delta \bar{a}}, \quad \text { if } \mathbf{w} \text { is above } \mathcal{L}^{U}
\end{aligned}
$$

and

$$
\begin{aligned}
\frac{\mathrm{d} \Delta \bar{a}}{\mathrm{~d} \Delta \bar{\theta}} & =-\frac{b}{P_{0} \Delta \bar{a}}\left(C_{B 0}^{*}\left(-\rho_{C}^{*} \zeta+\rho_{T}^{*}\right)\right) \\
& =-\frac{b C_{B 0}^{*} \rho_{T}^{*}\left(-\zeta\left(1-\frac{\Delta \bar{a}}{H}\right)+1\right)}{P_{0} \Delta \bar{a}}, \quad \text { if } \mathbf{w} \text { is below } \mathcal{L}^{L}
\end{aligned}
$$

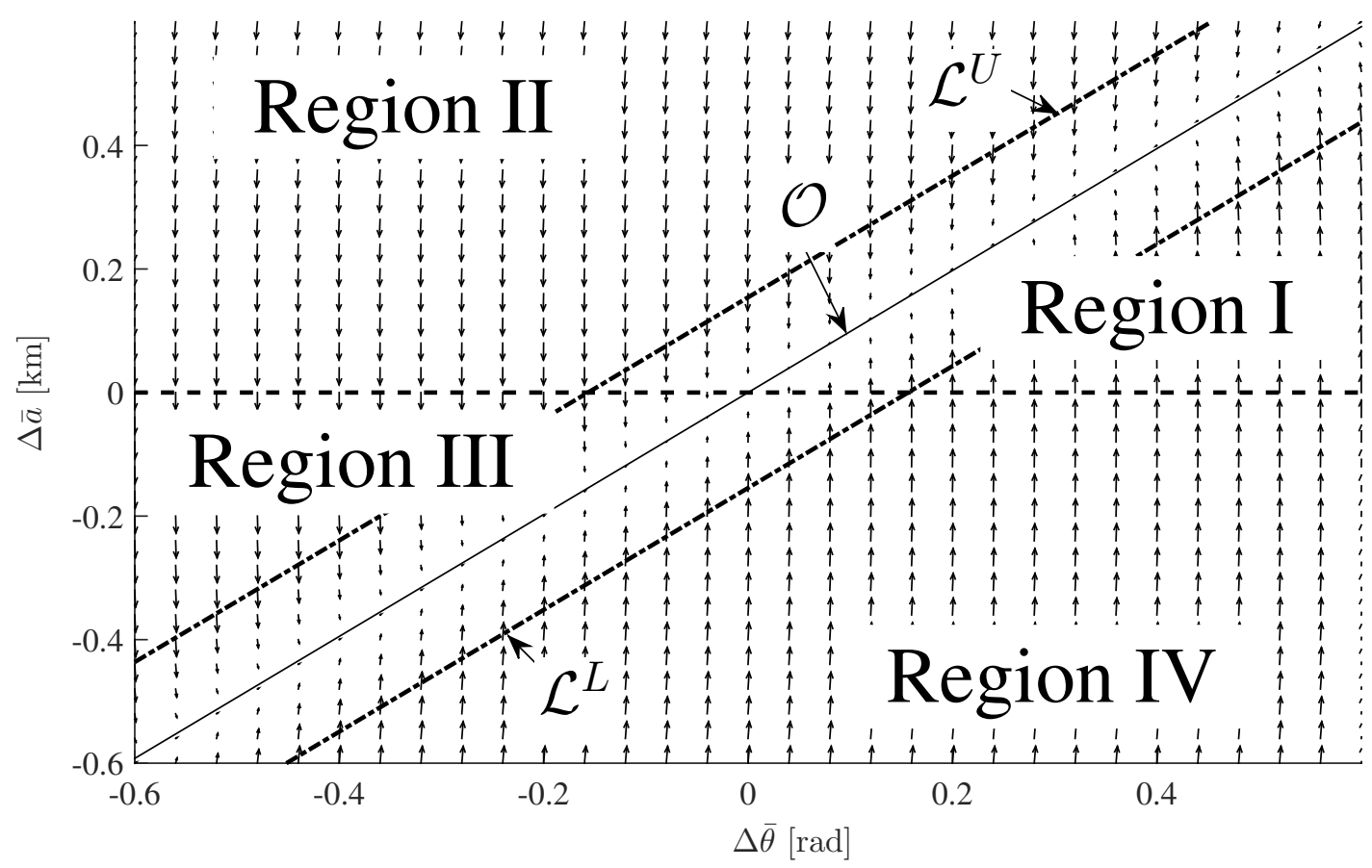

Fig. 2 Phase Portrait of the System, depicted with Eqs. (51-53).

The line defined as $\Delta \bar{a}=0$ along with the line $\mathcal{O}$ determine four regions of the phase portrait, 
each of which has a distinctive flow direction. These regions are named I, II, III, and IV. In region I, the flow has $\Delta \dot{\bar{a}}>0$ and $\Delta \dot{\bar{\theta}}<0$. In region II, the flow evolves such that $\Delta \dot{\bar{a}}<0$ and $\Delta \dot{\bar{\theta}}<0$. In region III, the flow is characterized by $\Delta \dot{\bar{a}}<0$ and $\Delta \dot{\bar{\theta}}>0$. Finally, in region IV, the flow moves with $\Delta \dot{\bar{a}}>0$ and $\Delta \dot{\bar{\theta}}>0$. Each of these regions has a sub-region within the non-saturated zone, denoted by $(\cdot)^{\mathcal{N}}$, and a sub-region that is outside the non-saturated zone, which is denoted by $(\cdot)^{\mathcal{S}}$. Hence, for instance, region I outside(inside) the non-saturated zone will be referred to as $\mathrm{I}^{\mathcal{S}(\mathcal{N})}$.

As time elapses, considering the slow decay of $\bar{a}_{T}$ and consequent growth of $\rho_{T}^{*}$, the slopes outside the non-saturated zone become steeper, as seen from Eqs. (52) and (53). Due to the flow directions of regions $\mathrm{I}^{\mathcal{S}}$ and $\mathrm{III}^{\mathcal{S}}$, the trajectories will eventually reach the non-saturated zone. In region $\mathrm{IV}^{\mathcal{S}}$, as long as

$$
\frac{\mathrm{d} \Delta \bar{a}}{\mathrm{~d} \Delta \bar{\theta}}>S_{L}
$$

the trajectories will reach either the region $\mathrm{IV}^{\mathcal{N}}$ or the region $\mathrm{I}^{\mathcal{S}}$, in which case they also end up at the non-saturated zone. Inequality (54), entails

$$
\Delta \bar{a}>\frac{\zeta-1}{\frac{P_{0} S_{L}}{b C_{B 0}^{*} \rho_{T}^{*}}+\frac{\zeta}{H}}
$$

and with the same typical values used to build Fig. 2 was built, inequality (54) is satisfied as long as $\Delta \bar{a}>-22.03 \mathrm{~km}$. In region $\mathrm{II}^{\mathcal{S}}$, as long as

$$
\frac{\mathrm{d} \Delta \bar{a}}{\mathrm{~d} \Delta \bar{\theta}}>S_{U}
$$

the trajectories will flow towards the region $\mathrm{II}^{\mathcal{N}}$ or $\mathrm{III}^{\mathcal{S}}$ in which case they will eventually reach the non-saturated zone as well. Condition (56) implies

$$
\Delta \bar{a}<\frac{1-\zeta}{\frac{P_{0} S_{U}}{b C_{B 0}^{*} \rho_{T}^{*}}+\frac{1}{H}}
$$

With the same values used to build Fig. 2, inequality (56) is satisfied as long as $\Delta \bar{a}<16.06 \mathrm{~km}$. 
For practical purposes and due to aforesaid reasons, the value of $|\Delta \bar{a}|$ is expected to be significantly smaller than $\sim 16 \mathrm{~km}$. Since the phase portrait shows that the trajectories always move towards and eventually enter the non-saturated zone, they cannot leave once they reached it. Therefore, once the trajectories are within the non-saturated zone, the LQR controller drives the system towards the origin, with no saturation.

Under the presence of uncertainties, the input of the system is given by Eq. (31). $\eta$ can actually affect the slopes of the flow directions. To measure these effects consider the following.

$$
\Delta \dot{\bar{a}}=b\left(\nu_{\text {des }}+\eta\right)
$$

while $\eta$ does not explicitly affect $\Delta \dot{\bar{\theta}}=-P_{0} \Delta \bar{a}$. As stated by Eq. (54), if in region $\mathrm{IV}^{\mathcal{S}}$ the slopes $\mathrm{d} \Delta \bar{a} / \mathrm{d} \Delta \bar{\theta}=\Delta \dot{\bar{a}} / \Delta \dot{\bar{\theta}}$ are higher than $S_{L}$, the system will eventually reach the non-saturated zone. Hence,

$$
\begin{gathered}
\Delta \dot{\bar{\theta}} S_{L}<\Delta \dot{\bar{a}} \\
-P_{0} \Delta \bar{a} S_{L}<b C_{B 0}^{*}\left(-\rho_{C}^{*} \zeta+\rho_{T}^{*}+\eta\right)
\end{gathered}
$$

for any $\eta$. If

$$
|\eta| \leq \bar{\eta}<\rho_{T}^{*}\left(1-\zeta\left(1-\frac{\Delta \bar{a}}{H}\right)\right)+\frac{P_{0} \Delta \bar{a} S_{L}}{b C_{B 0}^{*}}
$$

holds, as previously mentioned, the trajectories will reach either the region $\mathrm{IV}^{\mathcal{N}}$ or the region $\mathrm{I}^{\mathcal{S}}$, in which case they also end up at the non-saturated zone. For region $\mathrm{II}^{\mathcal{S}}$, since $\Delta \dot{\bar{\theta}}<0$,

$$
\begin{gathered}
\Delta \dot{\bar{\theta}} S_{U}>\Delta \dot{\bar{a}} \\
-P_{0} \Delta \overline{\bar{a}} S_{U}>b C_{B 0}^{*}\left(-\rho_{C}^{*}+\rho_{T}^{*} \zeta+\eta\right)
\end{gathered}
$$


for any $\eta$. If

$$
|\eta| \leq \bar{\eta}<\rho_{T}^{*}\left(1-\zeta-\frac{\Delta \bar{a}}{H}\right)-\frac{P_{0} \Delta \bar{a} S_{U}}{b C_{B 0}^{*}}
$$

holds, as previously mentioned, the trajectories will reach either the region $\mathrm{II}^{\mathcal{N}}$ or the region $\mathrm{III}^{\mathcal{S}}$, in which case they also end up at the non-saturated zone. Numerical values of the expressions involved in the inequalities will be shown in Section IV.

\section{E. Computation of $\beta_{\mathrm{C}}$ and $\beta_{\mathrm{T}}$}

Using Eqs. (40-42), the angles $\beta_{C}$ and $\beta_{T}$ are determined. If the system is within the nonsaturated zone, then $\beta_{C}$ and $\beta_{T}$ must satisfy Eq. (42), which constitutes a single equation with two unknowns. In order to minimize the orbital decay, it is sought that the cross-sectional areas are always as small as possible. Therefore, one of the angles may be arbitrarily set to yield the minimum possible cross-sectional area, and the other one determined to solve Eq. (42). Hence, the following algorithm is proposed to determine $\beta_{C}$ and $\beta_{T}$.

$$
\begin{cases}\beta_{C}=90^{\circ} \text { and } \beta_{T}=90^{\circ}, & \text { if } \bar{a}_{C}=\bar{a}_{T} \text { and } \bar{\theta}_{C}=\bar{\theta}_{T} \\ \beta_{C}=90^{\circ} \text { and } \beta_{T}=\arccos \left[\frac{-\mathbf{K w}^{\top}+\rho_{C}^{*} C_{B 0}^{*} \zeta}{C_{B 0}^{*} \rho_{T}^{*}}\right]+\psi, & \text { if }-\mathbf{K w} \geq 0 \\ \beta_{T}=90^{\circ} \text { and } \beta_{C}=\arccos \left[\frac{\mathbf{K w} \mathbf{w}^{\top}+\rho_{T}^{*} C_{B 0}^{*} \zeta}{C_{B 0}^{*} \rho_{C}^{*}}\right]+\psi, & \text { otherwise }\end{cases}
$$

\section{Numerical Simulations}

The developed control law was tested in a few simulations. The scenarios were built such that they include realistic effects that affect the dynamics of LEO satellites while maneuvering based on DD. Five simulations will be elaborated here. They will be referred to as Cases, and they are summarized in Table (1).

Every case implements the density model NRLMSISE-00 [31] as the true density field (hence giving $\rho_{C}$ and $\rho_{T}$ ), considering the corresponding variations of the F10.7 solar flux index as well as the geomagnetic index AP. To that end, the values of these indices were retrieved from NASA/GSFC's OMNI data base through OMNIWeb. The data were retrieved for the entire years 2009 through 
Table 1 Summary of the cases simulated

\begin{tabular}{cccccc}
\hline Case \# & $C_{D}$ & Co-rotation & Zonal Harmonics & $\bar{i}_{C / T}$ & Gains $\tilde{q}_{1}=\tilde{q}_{2}$ \\
\hline I & 2.39 & No & Up to $J_{2}$ & $10 \mathrm{deg}$ & $5 \cdot 10^{-17}$ \\
II & 2.39 & No & Up to $J_{2}$ & $97 \mathrm{deg}$ & $5 \cdot 10^{-17}$ \\
III & Eq. $(66)$ & No & Up to $J_{2}$ & $10 \mathrm{deg}$ & $5 \cdot 10^{-17}$ \\
IV & Eq. $(66)$ & $\mathbf{v}_{\text {atm }}=\boldsymbol{\Omega}_{\mathrm{E}} \times \mathbf{r}$ & Up to $J_{8}$ & $97 \mathrm{deg}$ & $5 \cdot 10^{-17}$ \\
V & Eq. $(66)$ & $\mathbf{v}_{\text {atm }}=\boldsymbol{\Omega}_{\mathrm{E}} \times \mathbf{r}$ & Up to $J_{8}$ & $97 \mathrm{deg}$ & $5 \cdot 10^{-19}$
\end{tabular}

2011. The presented simulations start on January $11^{t h}, 2010$, at 12:23:00 UT, and with the retrieved data, they cannot extend beyond December $31^{\text {st }}$, 2011, at 23:59:59 UT, since simulations with realistic indices were pursued. On the other hand, the density models that are assumed as known by the controllers, $\rho_{C}^{*}$ and $\rho_{T}^{*}$, were set as $\rho_{C}^{*}=\rho_{T}^{*}=1.1 \cdot 10^{-12} \mathrm{~kg} / \mathrm{m}^{3}$, which were arbitrarily set, according to the density behavior for 2009 as modeled by NRLMSISE-00. Notice that $\rho_{C}^{*}$ and $\rho_{T}^{*}$ could be also assumed to have some kind of variations. Whatever model is assumed for $\rho_{C}^{*}$ and $\rho_{T}^{*}$, the bound for the uncertainties $\delta \rho^{M}$ should be estimated accordingly, if an accurate computation of $\bar{\eta}$ is desired.

NRLMSISE-00 was also used to obtain the true temperature. These temperature values were used to implement models of drag coefficient variations, as will be elaborated in the following subsections.

For Cases I and II, the real drag coefficient $C_{D}$ was arbitrarily considered to be 2.39 and constant, whereas for other cases a more realistic model that accounts for the spacecraft attitude, the temperatures of the atmosphere's and surfaces of the spacecraft, as well as their chemical composition was simulated. For all the cases, the $C_{D}^{*}$ assumed by the controller was set as $C_{D}^{*}=2.2$, generating a realistic amount of uncertainty in this parameter. Moreover, the masses of the satellites were set as $5 \mathrm{~kg}$ each, while $S_{1}=0.06 \mathrm{~m}^{2}$ and $S_{2}=0.01 \mathrm{~m}^{2}$.

The simulations were run in cartesian elements, with the corresponding nonlinear differential equations. Since the theoretical developments consider the secular effects generated by the $J_{2}$ zonal harmonics, all the simulations include the corresponding $J_{2}$ terms in the equations of motion. Moreover, to assess dynamical effects not considered in the development of the LQR, Cases IV and $\mathrm{V}$ include zonal harmonics terms up to $8^{\text {th }}$ degree as well as co-rotation of the atmosphere, which add more uncertainties than those accounted for in the presented developments. 
The equations of motion integrated in the simulations are given by

$$
\ddot{\mathbf{r}}=\nabla \mathcal{R}-\frac{1}{2 m} \rho\left\|\mathbf{v}-\mathbf{v}_{\text {atm }}\right\|\left(\mathbf{v}-\mathbf{v}_{\text {atm }}\right) \sum_{i=1}^{3} A_{i} C_{D_{i}}
$$

where $A_{i}$ stands for the cross-sectional area of the surface $i$, and $C_{D_{i}}$ denotes the drag coefficient corresponding to the same surface, which in general will be considered as functions of the attitude. $\mathbf{v}_{\text {atm }}$ denotes the velocity of the atmosphere. For Cases I, II and III, it is assumed as $\mathbf{v}_{\text {atm }}=\mathbf{0}$, while for Cases IV and V it was assumed as $\mathbf{v}_{\text {atm }}=\boldsymbol{\Omega}_{\mathrm{E}} \times \mathbf{r}$, where $\boldsymbol{\Omega}_{\mathrm{E}}$ represents the angular velocity of the Earth in an Earth Centered Inertial frame (ECI), assumed to be $\boldsymbol{\Omega}_{\mathrm{E}}=[0,0,2 \pi / 86164.1]^{\top} \mathrm{rad} / \mathrm{s}$, and $\mathbf{r}=[x, y, z]^{\top}$ represents the position vector of the satellite in the ECI frame. $\mathcal{R}$ represents the considered geopotential including zonal harmonics and is given by [1, pp. 543]:

$$
\mathcal{R}=\frac{\mu}{r}\left[1-\sum_{l=2}^{L} J_{l}\left(\frac{R_{\mathrm{E}}}{r}\right)^{l} P_{l}\left(\frac{z}{r}\right)\right]
$$

and $\nabla$ the gradient operator taken in cartesian coordinates. Moreover, $r=\|\mathbf{r}\|$, and $P_{l}\left(\frac{z}{r}\right)$ denotes the Legendre polynomial of the first kind and order $l$, computed at the ratio $z / r$.

It is worth mentioning that in most works and simulations found in the literature, dealing with DD maneuvering, the effects due to variations of solar and geomagnetic indices, the co-rotation of the atmosphere, and the variation of the drag coefficient with the attitude and temperature are neglected. Even simulations performed with Systems Tool Kit (STK $\left.{ }^{\circledR}\right)$ using the High Precision Orbital Propagator (HPOP), tend to neglect some of these effects because HPOP considers a constant $C_{D}$

Since the controllers are formulated in terms of mean orbital elements, but the dynamic equations are formulated in cartesian elements, transformations from mean(osculating) orbital elements to osculating(mean) orbital elements should be implemented. To that end, this work implemented the First Order Approximation of the Brouwer theory [32], which for the purpose of this work is seen as a tool to convert from osculating(mean) elements to mean(osculating) representations.

In terms of mean elements, the considered initial conditions are given by $\bar{a}_{C}\left(t_{0}\right)=6800 \mathrm{~km}$, $\bar{e}_{C}\left(t_{0}\right)=0.0005, \bar{a}_{T}\left(t_{0}\right)=6800.01 \mathrm{~km}, \bar{e}_{T}\left(t_{0}\right)=0.001, \bar{\Omega}_{T}\left(t_{0}\right)=\bar{\Omega}_{C}\left(t_{0}\right)=0 \mathrm{deg}, \bar{\omega}_{T}\left(t_{0}\right)=$ 
$\bar{\omega}_{C}\left(t_{0}\right)=0 \mathrm{deg}$, and $\bar{M}_{T}\left(t_{0}\right)=20 \operatorname{deg} \bar{M}_{C}\left(t_{0}\right)=0 \mathrm{deg}$. Finally, the initial inclinations are specified for each simulation in Table (1). These initial conditions are transformed into the corresponding osculating counterparts, and then into cartesian elements, to be integrated. The gains utilized for the simulations were $\tilde{q}_{1}=\tilde{q}_{2}=5 \cdot 10^{-17}$, while $a_{0}=\bar{a}_{T}\left(t_{0}\right)$.

For the forthcoming simulations, the depicted results include the time history of $\Delta \bar{\theta}, \Delta \bar{a}, V$, $\beta_{C}$ and $\beta_{T}, \rho_{C}$ and $\rho_{T}, \rho_{C}^{*}$ and $\rho_{T}^{*}, \bar{a}_{C}$ and $\bar{a}_{T}$. Plots of the distance between the two satellites and out-of-plane coordinate of the relative position vector are shown for cases IV and V, in which co-rotation and $J_{8}$ are taken into account. In Cases I, II and III, the behaviors of the distance between the two satellites and the out-of-plane coordinate of the relative position vector were very similar to those shown.

\section{A. Numerical Estimation of the Uncertaity}

This section provides an estimation of the uncertainty on the model with respect to the reality defined for the simulations, based on Eq. (36). As previously stated, $\rho_{C}^{*}=\rho_{T}^{*}=1.1 \cdot 10^{-12} \mathrm{~kg} / \mathrm{m}^{3}$. Considering the assumed $\rho^{*}$ and according to retrieved data of density for the expected time of the maneuver, the $\delta \rho^{M}$ is considered as $\delta \rho^{M}=1.0 \cdot 10^{-12} \mathrm{~kg} / \mathrm{m}^{3}$, which represents about a $90 \%$ of error in the density model. According to Eq. (17), the maximum value for $u_{C}^{*}$ and $u_{T}^{*}$ can be stated as $u^{* M}=0.013382 \mathrm{~m}^{2} / \mathrm{kg}$. To estimate $\delta u^{M}$, with Eq. (2) and a model for $C_{D_{i}}$ given by Eq. (66), the variations of the ballistic coefficients as a function of the angle $\beta$ and temperature of the atmosphere was depicted. It is shown in Figure 3, which also includes the errors with respect to the modeled ballistic coefficient given by $C_{B 0} \cos (\beta-\psi)$.

From Fig. 3, it was determined that $\delta u^{M}=0.005223 \mathrm{~m}^{2} / \mathrm{kg}$. Finally, $\bar{\eta}=4.86966 \cdot 10^{-11}$ $1 / \mathrm{km}$. Recalling, the inequalities (60) and (62), considering $\Delta \bar{a}$ up to $4.9 \mathrm{~km}$ and with the values corresponding to the forthcoming simulations, the right hand sides of (60) and (62) attains values larger than $\bar{\eta}$, showing that under the expected uncertainties, the system will converge to the nonsaturated zone. Consider that the inequalities given by (60) and (62) are sufficient conditions, but not necessary. With the aforementioned values for $\tilde{q}_{1}$ and $\tilde{q}_{2}$, the value for expression (39) is obtained

as $\frac{\|\mathbf{P B}\|}{\lambda_{\min }}=2 \cdot 10^{8}$, which means that as long as the $\|\mathbf{w}\|>0.02, \dot{V}<0$. Recall that the involved 


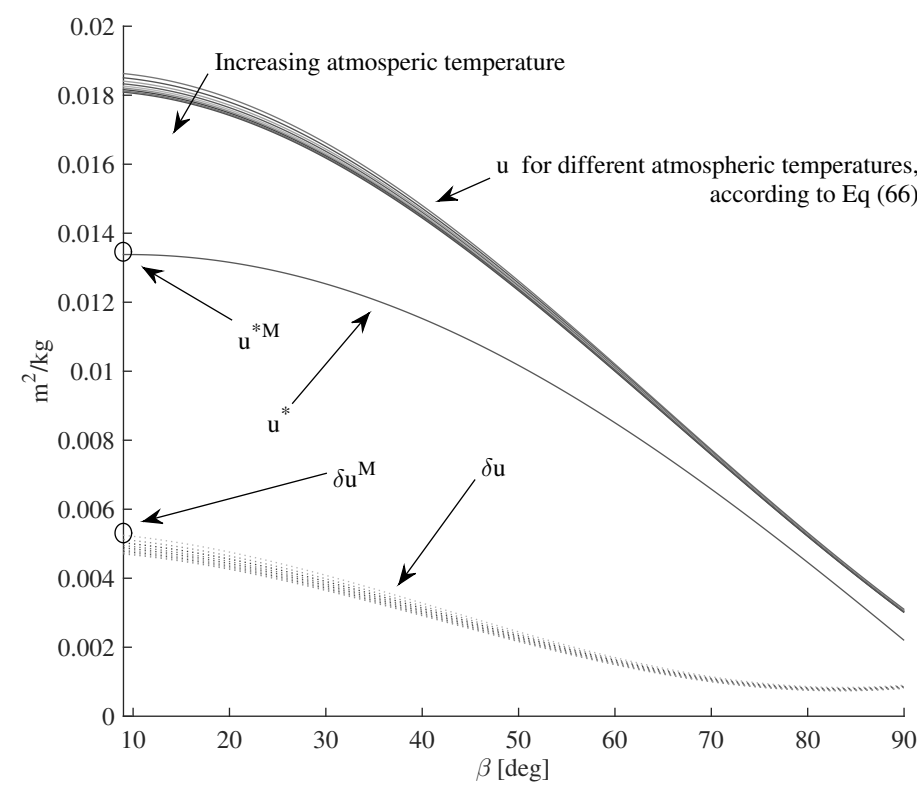

Fig. 3 Variation of $u$ and $\delta u$ with $\beta$ and atmospheric temperature ranging from 700K to $1500 \mathrm{~K}$.

quantities were adimensionalized using $1 \mathrm{~km}, 1 \mathrm{rad}, 1 \mathrm{~kg}$, and $1 \mathrm{sec}$.

\section{B. Case I and II}

The first two cases consider $C_{D_{i}}=2.39$ for every surface of both satellites. The initial mean inclination of Case I was set as $\bar{i}_{T}\left(t_{0}\right)=\bar{i}_{C}\left(t_{0}\right)=10 \mathrm{deg}$, while for Case II it was set as $\bar{i}_{T}\left(t_{0}\right)=$ $\bar{i}_{C}\left(t_{0}\right)=97 \mathrm{deg}$. Since DD cannot exert controlled out-of-plane differential specific forces, the simulations are always initiated with same orbital planes for Chaser and Target.

For these cases, the parameter $L$ of Eq. (65) was set as 2, so it only considers the $J_{2}$ zonal harmonic.

The results for Case I are depicted in Figures 4 and 5, while for Case II they are plotted in Figures 6 and 7 . The final distance between the two satellites were $25.12 \mathrm{~km}$ and $6.047 \mathrm{~km}$ for cases I and II, respectively, while the out-of-plane coordinate oscillated between $\pm 1.28 \mathrm{~km}$ and $\pm 0.90 \mathrm{~km}$ for cases I and II, respectively.

Figures 4 and 6 show the convergence of the maneuver, as expected from the analysis performed in the previous sections. However, the behaviors of the angles $\beta$ look different. Case I presents less oscillations in $\beta_{C}$ and $\beta_{T}$ than Case II. Examining the plots of the densities (Figs. 5 and 7 - middle plot), it is observed that the differences between the real values of density and the values assumed for 

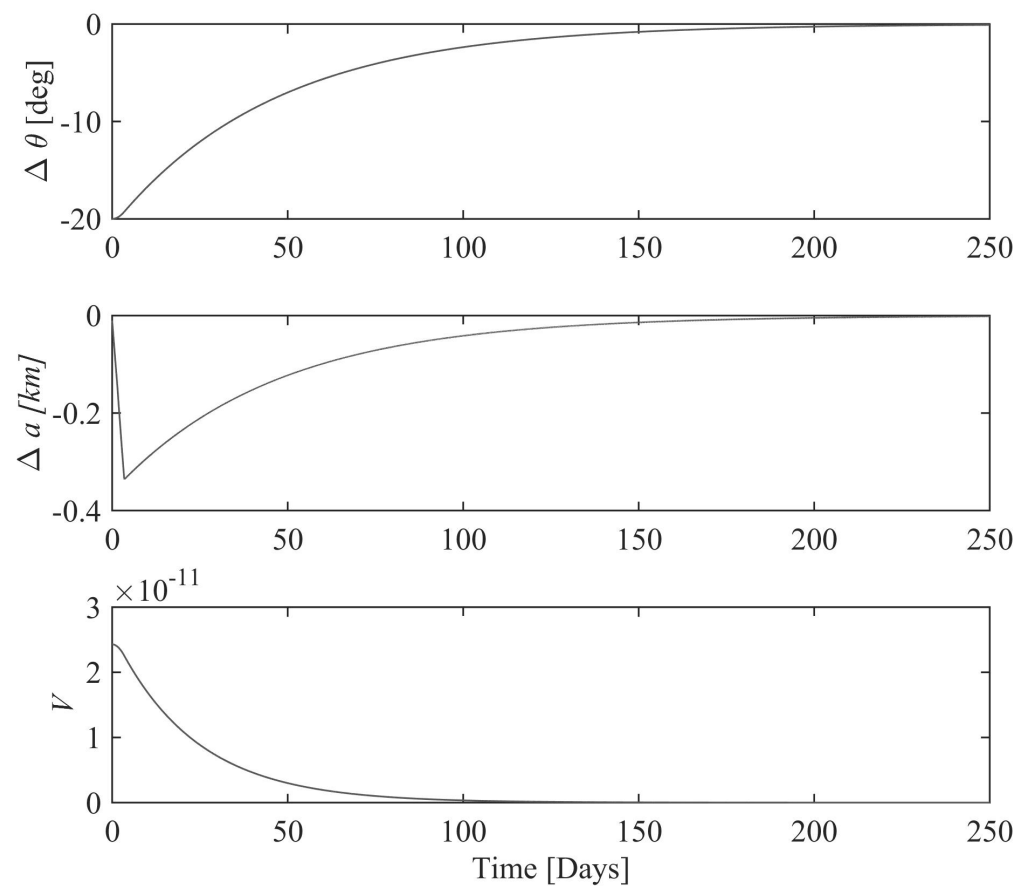

Fig. 4 Re-phasing Maneuver - Case I: $\Delta \bar{\theta}, \Delta \bar{a}$, and $V$.
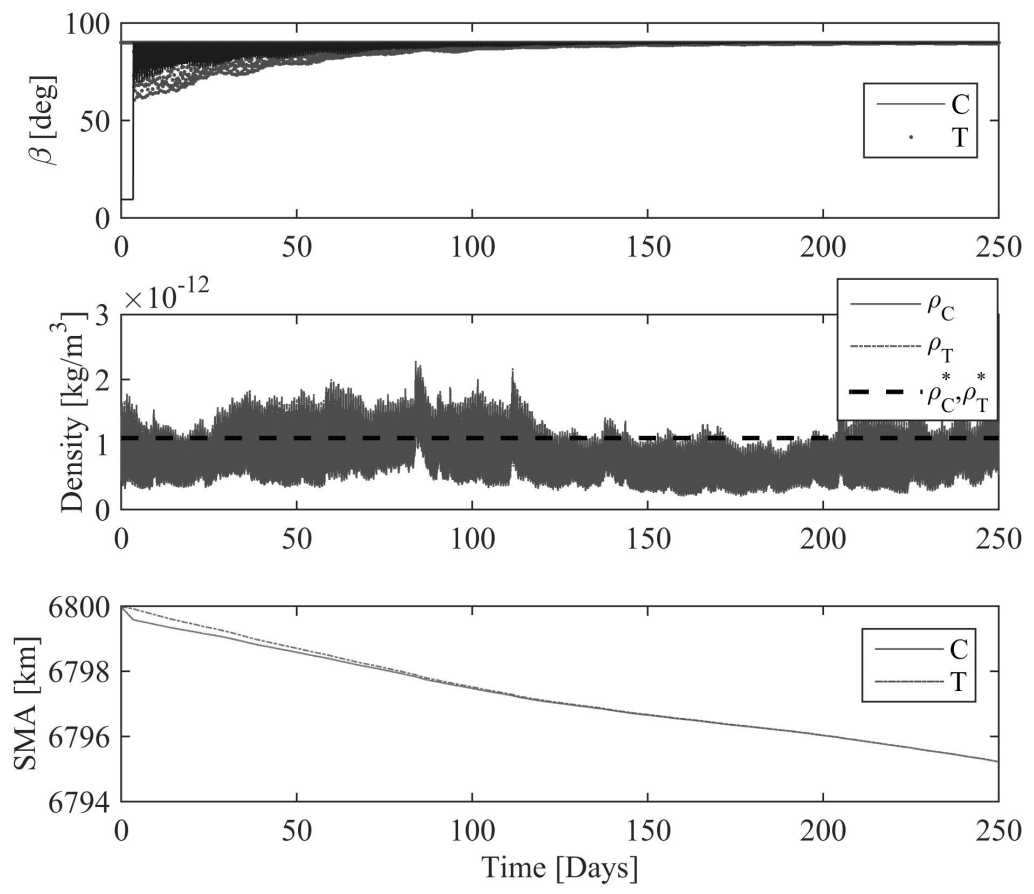

Fig. 5 Re-phasing Maneuver - Case I: $\beta, \rho$, and $\bar{a}$.

tuning the controllers, $\delta \rho$, are larger for Case II, reaching differences (uncertainties) of $90 \%$ during 

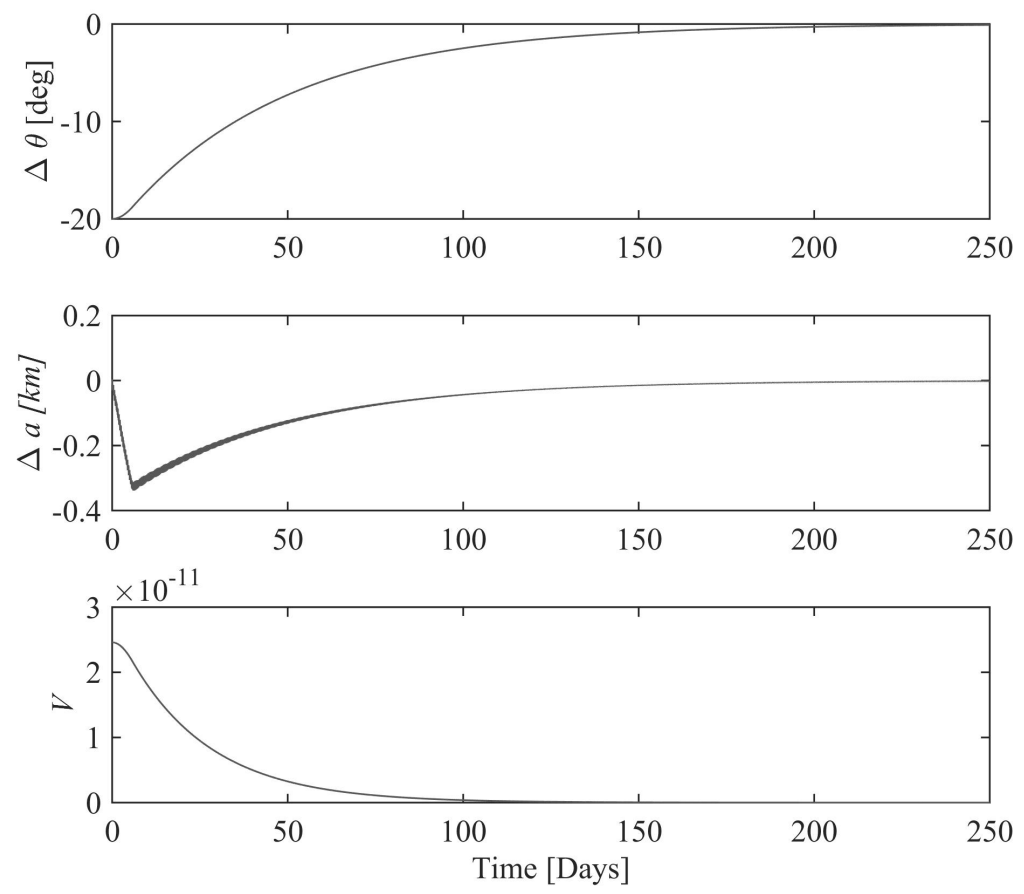

Fig. 6 Re-phasing Maneuver - Case II: $\Delta \bar{\theta}, \Delta \bar{a}$, and $V$.
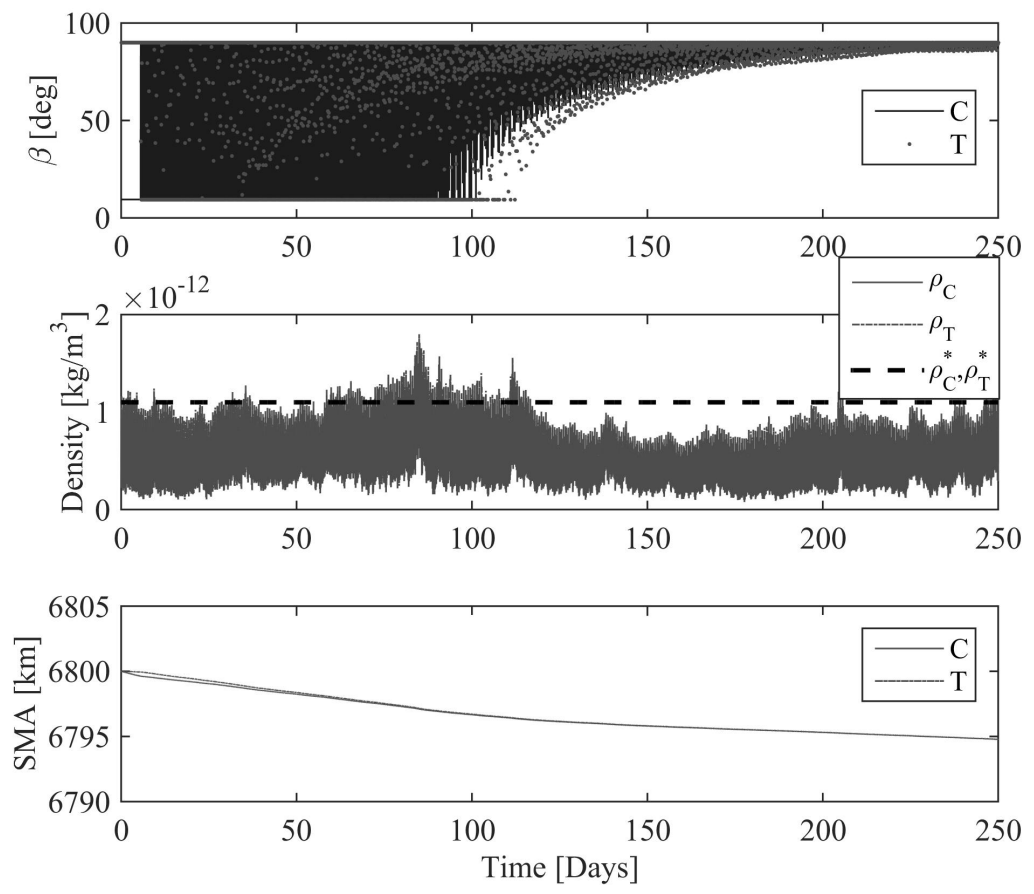

Fig. 7 Re-phasing Maneuver - Case II: $\beta, \rho$, and $\bar{a}$.

most of the maneuvering time, while for Case I these differences are not so accentuated. These 
differences are believed to cause the oscillations in the angles $\beta$, which in Case II vary between the extremes of the interval for almost 100 days, to overcome the high level of uncertainty in the input. If this constituted a difficulty for implementation, improving the models of the density used for the controller $\rho_{C}^{*}$ and $\rho_{T}^{*}$, or fine tuning of the LQR gains can help resulting in a smoother action. Although, the latter alternative could enlarge the region around the origin in which stability cannot be guaranteed. Another alternative would be to change the ballistic coefficient by other means than pitching the spacecraft, like for example rotating a set of panels, as the ORBCOMM satellites do [19].

From Figs. 5 and 7 , it is appreciated that the mean semi-major axes, $\bar{a}_{C}$ and $\bar{a}_{T}$, undergo a decay of $5 \mathrm{~km}$ approximately, validating the linearization proposed in Section III A.

\section{Case III}

The main purpose of this case is to introduce a more realistic model of the drag coefficient in the assumed true dynamics, which varies with the attitude and temperature. In fact, Case III differs from Case I only in the manner that the real drag coefficient is modeled. Still, $C_{D}^{*}$ assumed by the controller was set as $C_{D}^{*}=2.2$. The same initial inclination as Case I was considered, i.e. $\bar{i}_{T}\left(t_{0}\right)=\bar{i}_{C}\left(t_{0}\right)=10 \mathrm{deg}$.

Based on the works [26], [27] and [28], the drag coefficient of each surface is modeled as:

$$
C_{D_{i}}=2\left(1+\frac{2}{3} \sqrt{1+\alpha^{i}\left(\frac{T_{\mathrm{sat}}^{i}}{T_{\mathrm{atm}}}-1\right)} \sin \phi_{i}\right)
$$

where

$$
\alpha^{i}=\frac{3.6 u^{i}}{\left(1+u^{i}\right)^{2}}
$$

$T_{\text {sat }}^{i}$ denotes the temperature of the satellite surface $i, T_{\text {atm }}$ represents the kinetic temperature of the ambient gas molecules, and $u_{i}$ is the ratio of the mean mass of the incident gas atom to the mass of the surface atom of the surface $i$. For the purposes of this work, the aforementioned parameters were set as $T_{\text {sat }}^{i}=273 \mathrm{~K}$ (as done in [27] and [28]), and the temperature $T_{\text {atm }}$ was obtained from 
the NRLMSISE-00 model, with the corresponding F10.7 and AP indices. $\phi_{i}$ represents the angle of attack of the $i^{\text {th }}$ surface, which in fact varies with $\beta$. Finally, $u_{i}=0.215$ was arbitrarily set, assuming mean mass of the incident gas atom of 15 (nitrogen and oxygen atoms) and a mean mass of the atoms of the satellite surface to be 69.7 , corresponding to Gallium solar panels.

The results obtained from this simulation are depicted in Figures 8 and 9. The obtained behaviors look very similar to those corresponding to Case I, suggesting that the performance of the controller is not significantly affected by the incorporation of a more realistic model of the true $C_{D}$ behavior, which varies with the attitude and temperature. The final distance between the two satellites was $22.59 \mathrm{~km}$ and the out-of-plane coordinate oscillated between $\pm 1.28 \mathrm{~km}$.
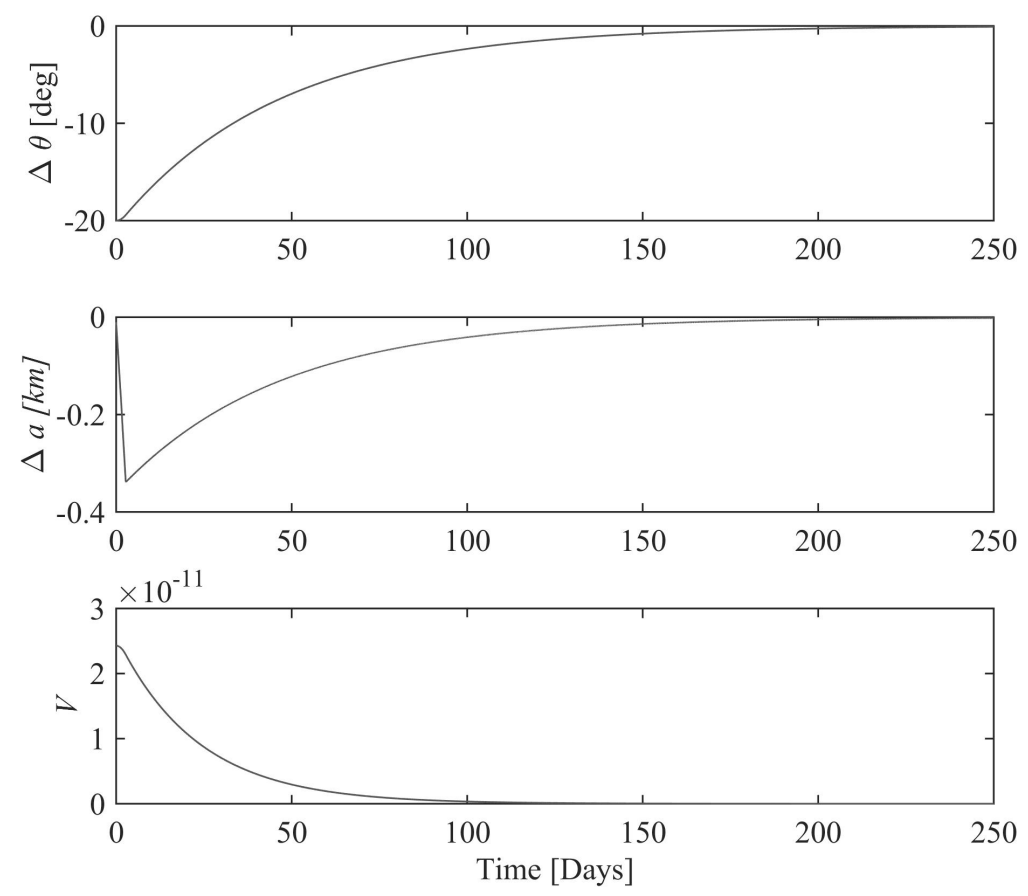

Fig. 8 Re-phasing Maneuver - Case III: $\Delta \bar{\theta}, \Delta \bar{a}$, and $V$.

\section{Case IV}

The purpose of this simulation is to assess the effects of the co-rotational motion of the atmosphere and Zonal harmonics including up to $8^{\text {th }}$ degree. Hence, The co-rotation is added by considering $\mathbf{v}_{\text {atm }}=\boldsymbol{\Omega}_{\mathrm{E}} \times \mathbf{r}$, where $\boldsymbol{\Omega}_{\mathrm{E}}=[0,0,2 \pi / 86164.1]^{\top} \mathrm{rad} / \mathrm{s}$. The initial inclination was set as $\bar{i}_{T}\left(t_{0}\right)=\bar{i}_{C}\left(t_{0}\right)=97 \mathrm{deg}$. 

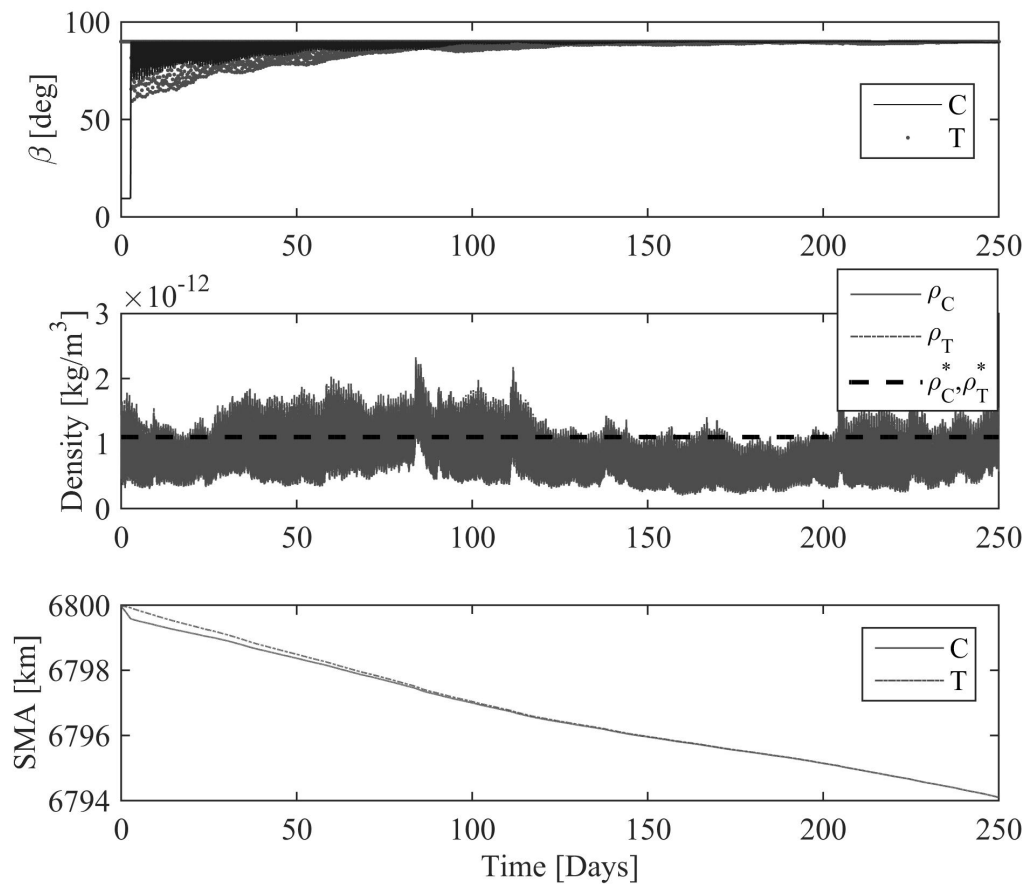

Fig. 9 Re-phasing Maneuver - Case III: $\beta, \rho$, and $\bar{a}$.

Since co-rotational motion of the atmosphere is considered, and the pitch axis is perpendicular to the plane of motion, it is necessary to consider the drag generated by the impinging particles of the atmosphere onto the lateral faces of the satellites. Hence, there will be an out-of-plane drag force, corresponding to the components of the vector $\mathbf{v}-\boldsymbol{\Omega}_{\mathrm{atm}} \times \mathbf{r}$ perpendicular to $\mathbf{v}$. The surface of each lateral face of the satellite was assumed $S_{3}=0.06 \mathrm{~m}^{2}$, like $S_{1}$. Furthermore, the variations of the drag coefficients with attitude and temperature are modeled, while $C_{D}^{*}=2.2$.

The results of the simulation are shown in Figures 10,11 and 12. it is observed that adding the co-rotation and more terms of the zonal harmonics series to the real dynamics, actually generates larger oscillations of the angles $\beta_{C}$ and $\beta_{T}$, which seems to be required to counteract the effects due to the added perturbations. This effect can be also associated to the implementation of the first order model of the Brouwer transformation, which only consider first order $J_{2}$ terms. Hence, it may not be able to remove oscillations in the orbital elements caused by higher zonal harmonics, which make the angles $\beta$ to increase their oscillations. Fine tuning of the LQR gain can help to reduce these oscillations. Nonetheless, under the level of uncertainty in the density and drag coefficients, and the unconsidered perturbations in the dynamical models, the controller still performs satisfactorily, 
driving the two satellites to the expected encounter.
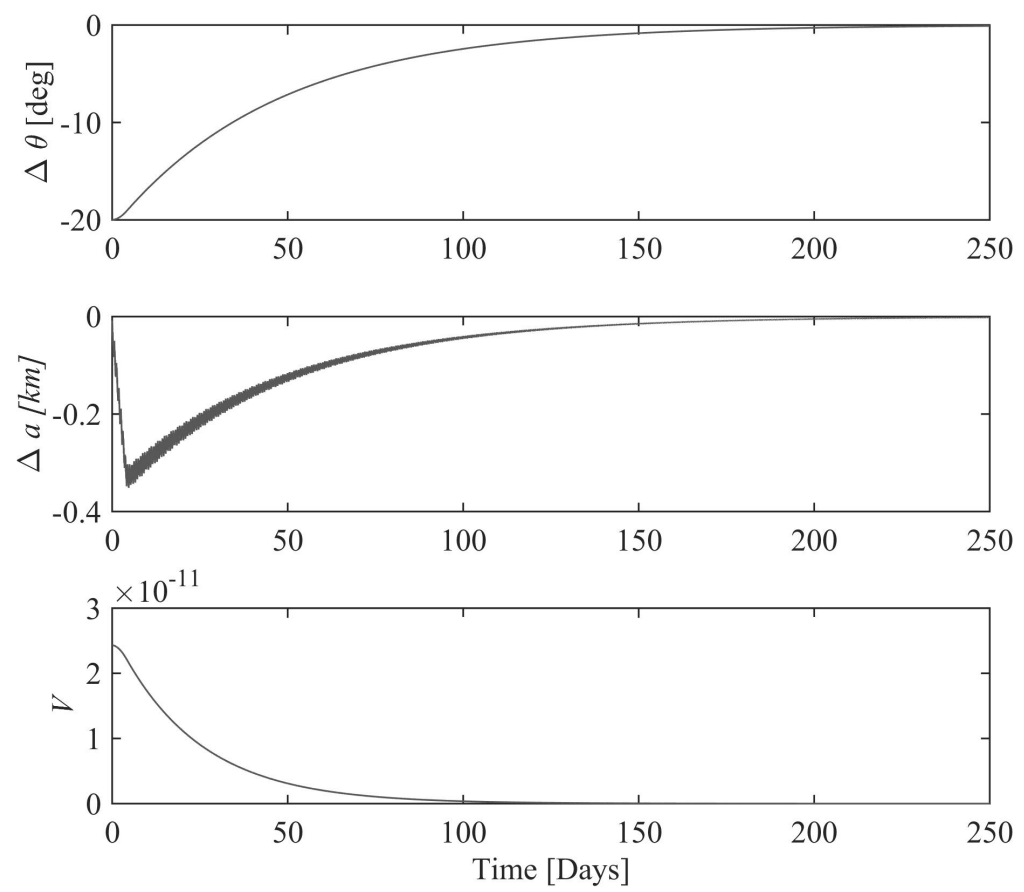

Fig. 10 Re-phasing Maneuver - Case IV: $\Delta \bar{\theta}, \Delta \bar{a}$, and $V$.
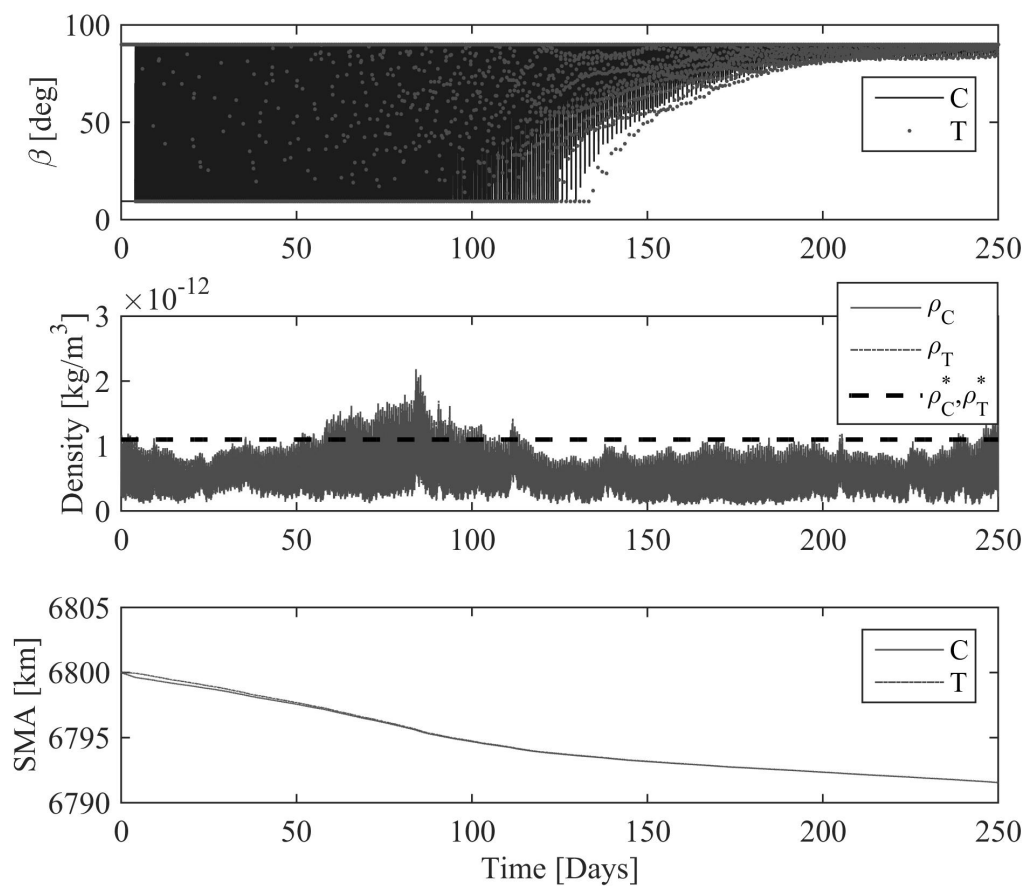

Fig. 11 Re-phasing Maneuver - Case IV: $\beta, \rho$, and $\bar{a}$. 


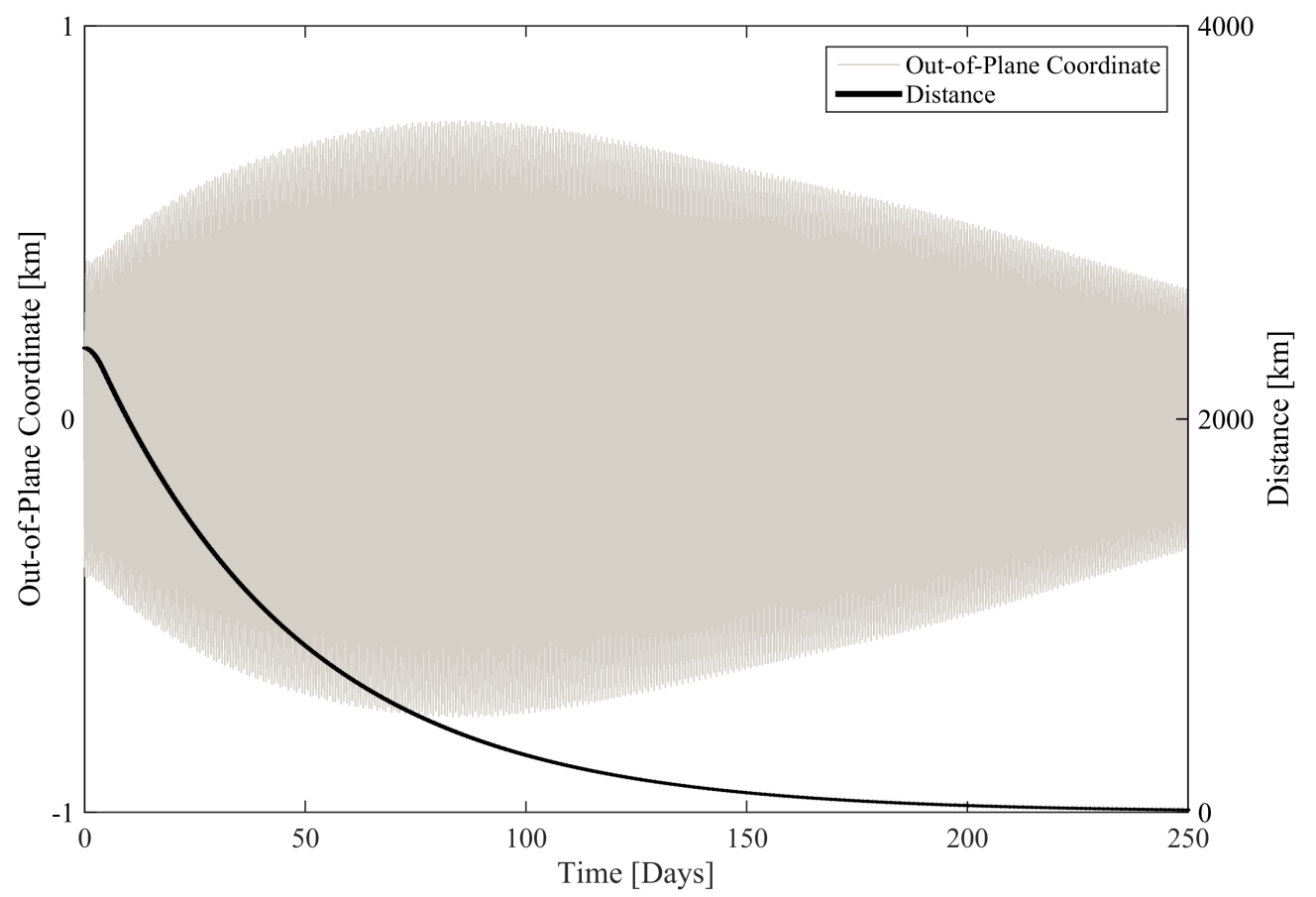

Fig. 12 Re-phasing Maneuver - Case IV: Inter-Satellite Distances and out-of-plane coordinate.

\section{E. Case V}

The purpose of this Case is to reduce the oscillations of the angles $\beta_{C}$ and $\beta_{T}$ by utilizing smaller gains for the LQR. In this Case $\tilde{q}_{1}=\tilde{q}_{2}$ were set to have a value of $5 \cdot 10^{-19}$ while for all the previous case they had a value of $5 \cdot 10^{-17}$. Otherwise this Case is identical to Case IV.

The results of these simulations are shown in Figures 13, 14, and 15. Comparing Figures 11 to 14, it is observed that the smaller LQR gains results in a faster reduction of the oscillation amplitude of the angles $\beta_{C}$ and $\beta_{T}$, although the size of the region where $\dot{V}<0$ cannot be ensured is enlarged. Similarly to all the other Cases, Case V shows that under the level of uncertainty in the density and drag coefficients, and the unconsidered perturbations in the dynamical models, the controller also performs satisfactorily, driving the two satellites to the expected encounter. 

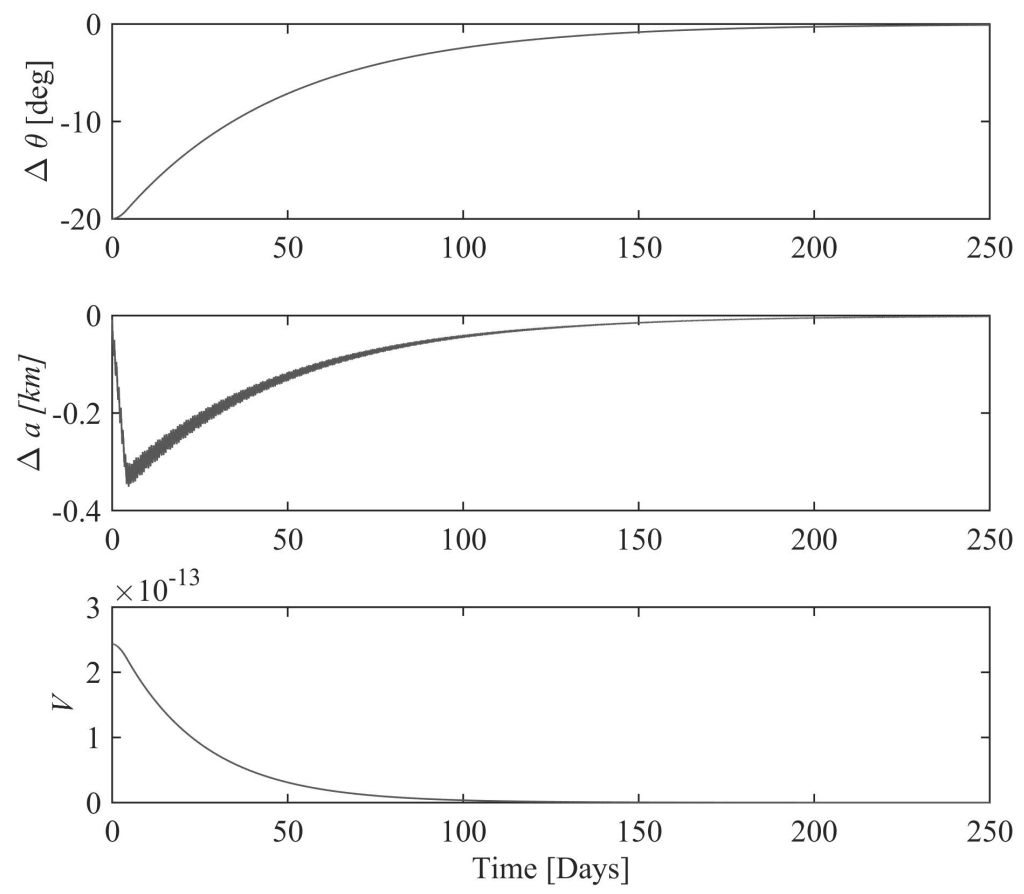

Fig. 13 Re-phasing Maneuver - Case V: $\Delta \bar{\theta}, \Delta \bar{a}$, and $V$.
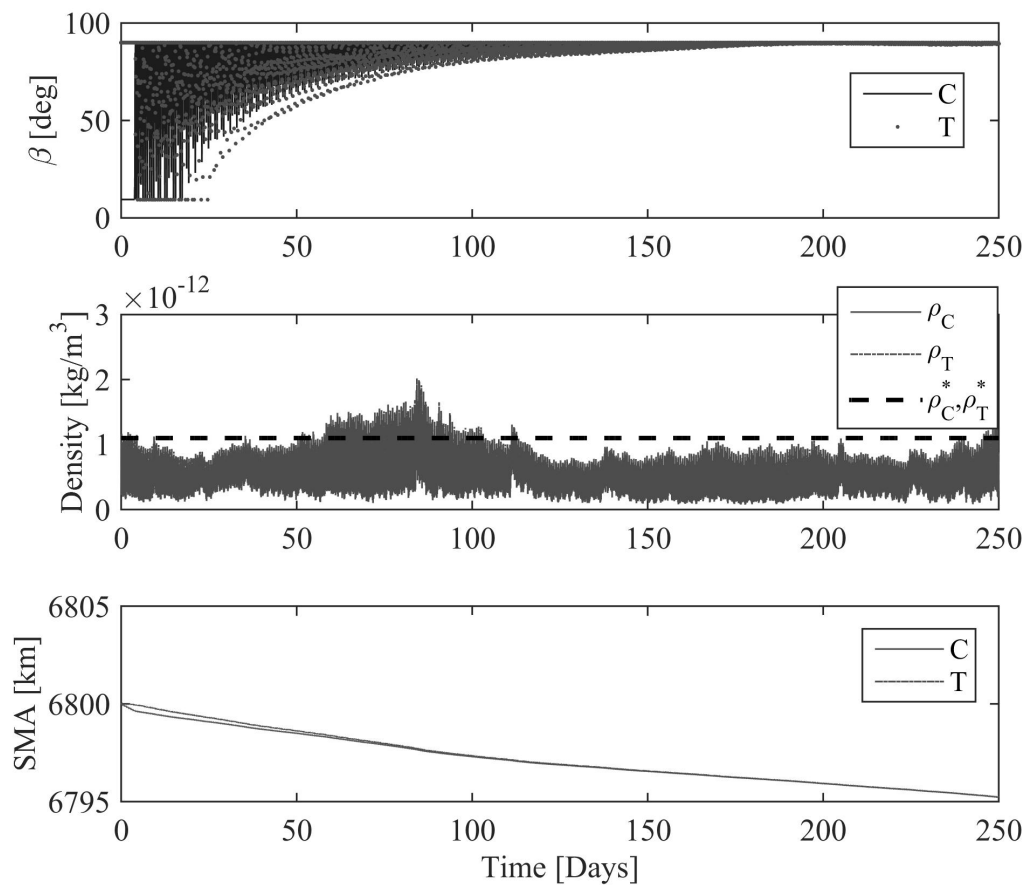

Fig. 14 Re-phasing Maneuver - Case V: $\beta, \rho$, and $\bar{a}$. 


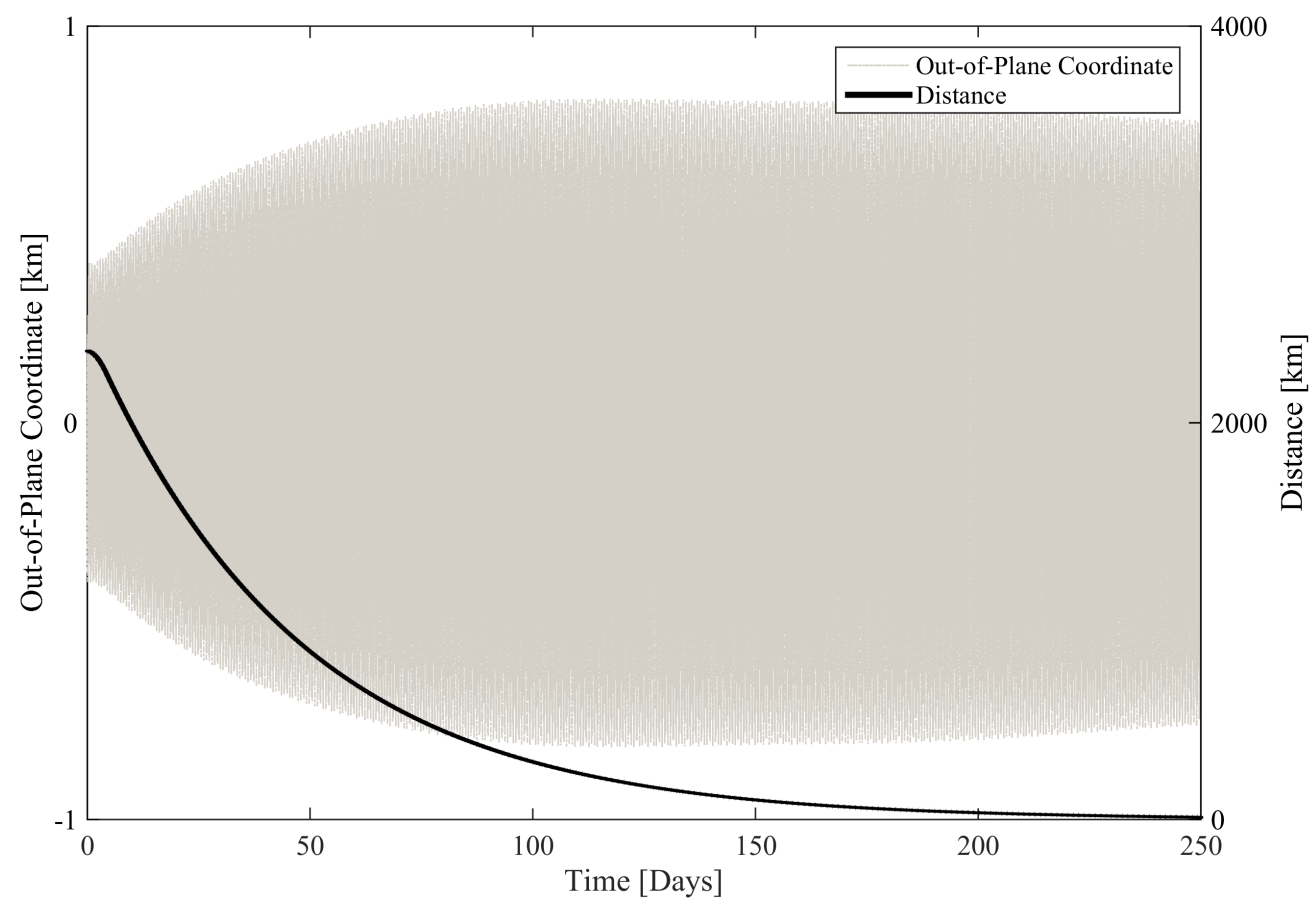

Fig. 15 Re-phasing Maneuver - Case V: Inter-Satellite Distances and out-of-plane coordinate.

\section{Conclusions}

In the last decades the idea of propellantless spacecraft relative maneuvering via differential drag has received substantial attention. Nevertheless, the explicit inclusion of drag model uncertainties in the control laws has been mostly neglected. This work presented a methodology to perform differential drag relative maneuvering of coplanar spacecraft, towards rendezvous, under bounded uncertainties in the drag force. The required differential drag accelerations were obtained by varying the pitch angles of the satellites, thus changing their ballistic coefficients.

The developed approach enables to consider long-range maneuvers, assuming that both satellite are initially in circular orbits. The developed dynamical system, based on mean semi-major axes and mean arguments of latitude, allows for the implementation of an Linear Quadratic Regulator with a saturation function. In presence of bounded uncertainties, convergence of the trajectories can be proved up to a certain norm of the state vector, for which an analytical expression was provided in terms of the uncertainties. The effect of saturation in the control was examined by analyzing the phase portrait of the system, which showed that the system will eventually desaturate, and hence converge. 
Several simulations were presented, including realistic effects like variation of the drag coefficients with the attitude and temperature, density field given by NRLMSISE-00 with realistic solar flux and geomagnetic indices behavior and co-rotation of the atmosphere and zonal harmonics. The simulations consistently show convergence of the maneuvers, illustrating the robustness of the approach under actual uncertainties and modeling errors, supporting the analytical developments.

An interesting natural extension of this work would consider lift forces and three-dimensional attitude variations, to examine the possibility of controlling the out-of-plane relative motion of the satellites, which could also be relevant to maneuver spacecraft that lose the propulsion system due to failures and/or propellant depletion. 


\section{Acknowledgments}

The authors wish to thank the University of Florida and the U.S. Air Force Office of Scientific Research (contract \# N00014-15-1-2087) for supporting this research. This work was initiated during Dr. Curti's visit at the University of Florida, in spring 2015.

\section{References}

[1] Vallado, D. A., Fundamentals of Astrodynamics and Applications, McGraw-Hill Inc., 2007.

[2] Finley, T., Rose, D., Nave, K., Wells, W., Redfern, J., Rose, R., and Ruf, C., "Techniques for LEO Constellation Deployment and Phasing Utilizing Differential Aerodynamic Drag," in "AIAA Astrodynamics Conference," , 2013.

[3] Kumar, B. S. and Ng, A., "A Bang-Bang Control Approach to Maneuver Spacecraft in a Formation with Differential Drag," in "Proceedings of the AIAA Guidance, Navigation and Control Conference and Exhibit,", 2008, http://dx.doi.org/10.2514/6.2008-6469.

[4] Leonard, C., Formationkeeping of Spacecraft Via Differential Drag, Master's thesis, Massachusetts Inst. of Technology, July 1986.

[5] Leonard, C., Hollister, W., and Bergmann, E., "Orbital formationkeeping with differential drag," Journal of Guidance, Control, and Dynamics, Vol. 12, No. 1, 1989, http://dx.doi.org/10.2514/3.20374, pp. 108-113.

[6] Clohessy, W. H. and Wiltshire, R. S., "Terminal guidance system for satellite rendezvous," Journal of the Aerospace Sciences, Vol. 27, No. 9, 1960, http://dx.doi.org/10.2514/8.8704, pp. 653-658.

[7] Carter, T. and Humi, M., "Clohessy-Wiltshire Equations Modified to Include Quadratic Drag," Journal of Guidance, Control, and Dynamics, Vol. 25, No. 6, 2002, http://dx.doi.org/10.2514/2.5010, pp. $1058-1063$.

[8] Bevilacqua, R. and Romano, M., "Rendezvous Maneuvers of Multiple Spacecraft using Differential Drag under J2 Perturbation," Journal of Guidance, Control, and Dynamics, Vol. 31, No. 6, 2008, http://dx.doi.org/10.2514/1.36362, pp. 1595-1607.

[9] Bevilacqua, R., Hall, J. S., and Romano, M., "Multiple spacecraft rendezvous maneuvers by differential drag and low thrust engines," Celestial Mechanics and Dynamical Astronomy, Vol. 106, No. 1, 2010, http://dx.doi.org/10.1007/s10569-009-9240-3, pp. 69-88.

[10] Schweighart, S. A. and Sedwick, R. J., "High-Fidelity Linearized J Model for Satellite Formation Flight," Journal of Guidance, Control, and Dynamics, Vol. 25, No. 6, 2002, http://dx.doi.org/10.2514/2. 4986, pp. 1073-1080. 
[11] Pérez, D. and Bevilacqua, R., "Differential drag spacecraft rendezvous using an adaptive Lyapunov control strategy," Acta Astronautica, Vol. 83, 2013, http://dx.doi.org/10.1016/j.actaastro.2012. 09.005, pp. 196-207.

[12] Ben-Yaacov, O. and Gurfil, P., "Long-Term Cluster Flight of Multiple Satellites using Differential Drag," Journal of Guidance, Control, and Dynamics, Vol. 36, No. 6, 2013, http://dx.doi.org/10.2514/1. 61496, pp. 1731-1740.

[13] Ben-Yaacov, O. and Gurfil, P., "Stability and Performance of Orbital Elements Feedback for Cluster Keeping Using Differential Drag," The Journal of the Astronautical Sciences, pp. 1-29.

[14] Schaub, H., "Incorporating secular drifts into the orbit element difference description of relative orbits," Advances in the Astronautical Sciences, Vol. 114, 2003, pp. 239-257.

[15] Harris, M. W. and Açıkmeşe, B., "Minimum time rendezvous of multiple spacecraft using differential drag," Journal of Guidance, Control, and Dynamics, Vol. 37, No. 2, 2014, http://dx.doi.org/10. 2514/1.61505, pp. 365-373.

[16] Dell'Elce, L. and Kerschen, G., "Comparison between analytical and optimal control techniques in the differential drag based rendez-vous," in "Proceedings of the 5th International Conference on Spacecraft Formation Flying Missions \& Technologies," , 2013.

[17] Dell'Elce, L., Martinusi, V., and Kerschen, G., "Robust optimal rendezvous using differential drag," in “Astrodynamics Specialist Conference,", 2014, http://dx.doi.org/10.2514/6.2014-4161.

[18] Dell'Elce, L., Satellite Orbits in the Atmosphere: Uncertainty Quantification, Propagation and Optimal Control, Ph.D. thesis, Univesité de Liège, 2015.

[19] Tuttle, C., "Satellite Stationkeeping of the ORBCOMM Constellation Via Active Control of Atmospheric Drag: Operations, Constraints, and Performance (AAS 05-152)," .

[20] Vallado, D. A. and Finkleman, D., "A critical assessment of satellite drag and atmospheric density modeling," Acta Astronautica, Vol. 95, 2014, http://dx.doi.org/10.1016/j.actaastro.2013.10.005, pp. $141-165$.

[21] Mishne, D., "Formation Control of Satellites Subject to Drag Variations and $J_{2}$ Perturbations," Journal of Guidance, Control and Dynamics, Vol. 27, No. 4, 2004, http://dx.doi.org/10.2514/1.11156, pp. $685-692$.

[22] Schaub, H., Vadali, S. R., Junkins, J. L., and Alfriend, K. T., "Spacecraft Formation Flying Control using Mean Orbit Elements," Journal of the Astronautical Sciences, Vol. 48, No. 1, 2000, pp. 69-87.

[23] Schaub, H. and Alfriend, K. T., "Impulsive Feedback Control to Establish Specific Mean Orbit Elements of Spacecraft Formations," Journal of Guidance, Control, and Dynamics, Vol. 24, No. 4, 2001, pp. 739- 
745.

[24] Mazal, L. and Gurfil, P., "Closed-Loop Distance-Keeping for Long-Term Satellite Cluster Flight," Acta Astronautica, Vol. 94, No. 1, 2014, http://dx.doi.org/10.1016/j.actaastro.2013.08.002, pp. 7382.

[25] Battin, R., An Introduction to the Mathematics and Methods of Astrodynamics, AIAA Education Series, 1999, http://dx.doi.org/10.2514/4.861543.

[26] Cook, G., "Satellite drag coefficients," Planetary and Space Science, Vol. 13, No. 10, 1965, pp. 929-946.

[27] Bruinsma, S., Tamagnan, D., and Biancale, R., "Atmospheric densities derived from CHAMP/STAR accelerometer observations," Planetary and Space Science, Vol. 52, No. 4, 2004, pp. 297-312.

[28] Sutton, E. K., Nerem, R. S., and Forbes, J. M., "Density and winds in the thermosphere deduced from accelerometer data," Journal of Spacecraft and Rockets, Vol. 44, No. 6, 2007, pp. 1210-1219.

[29] Kwakernaak, H. and Sivan, R., Linear optimal control systems, Vol. 1, Wiley-interscience New York, 1972.

[30] Khalil, H. K., Nonlinear systems, Prentice Hall, New Jersey, 2000.

[31] Picone, J., Hedin, A., Drob, D. P., and Aikin, A., "NRLMSISE-00 empirical model of the atmosphere: Statistical comparisons and scientific issues," Journal of Geophysical Research: Space Physics (19782012), Vol. 107, No. A12, 2002, pp. SIA-15.

[32] Schaub, H. and Junkins, J., Analytical Mechanics of Aerospace systems, Vol. 1, AIAA, 2003, Appendix F, pp. 693-696. 